\title{
One-Sided Synthetic control charts for monitoring the Multivariate Coefficient of Variation
}

\author{
Q. T. Nguyen ${ }^{\mathrm{a}}$, K. P. Tran ${ }^{\mathrm{b}}$, P. Castagliola ${ }^{\mathrm{c}}$, G. Celano $^{\mathrm{d}}$ and S. Lardjane ${ }^{\mathrm{a}}$ \\ ${ }^{a}$ Université de Bretagne-Sud \& LMBA UMR CNRS 6205, Vannes, France, ${ }^{\text {b}}$ Ecole Nationale \\ Supérieure des Arts et Industries Textiles, GEMTEX Laboratory, BP 30329, 59056 Roubaix \\ Cedex 1, France; ${ }^{\mathrm{c} U n i v e r s i t e ́ ~ d e ~ N a n t e s ~ \& ~ L S 2 N ~ U M R ~ C N R S ~ 6004, ~ N a n t e s, ~ F r a n c e ; ~}$ \\ ${ }^{\mathrm{d}}$ Università di Catania, Catania, Italy
}

\begin{abstract}
Shewhart's type control charts for monitoring the Multivariate Coefficient of Variation $(\mathrm{MCV})$ have recently been proposed in order to monitor the relative variability compared with the mean. These approaches are known to be rather slow in the detection of small or moderate process shifts. In this paper, in order to improve the detection efficiency, two one-sided Synthetic charts for the MCV are proposed. A Markov chain method is used to evaluate the statistical performance of the proposed charts. Furthermore, computational experiments reveal that the proposed control charts outperform the Shewhart MCV control chart in terms of the average run length to detect an out-of-control state. Finally, the implementation of the proposed chart is illustrated with an example using steel sleeves data.
\end{abstract}

\section{KEYWORDS}

Synthetic chart; Markov chain; Multivariate Coefficient of Variation; Steady-state

\section{Introduction}

Control charts are the most widely used Statistical Process Control tool. Under the usual assumptions, when implementing a control chart, the aim of quality practitioners is showing that the process position and/or scale are independently stable, to declare the in-control state for a process. However, in some processes, the location and scale are not independent of each other, that is, the population variance $\sigma^{2}$ is a function of the mean $\mu$. In these situations, the process is assumed to be in the in-control state when a parameter defined as a function of both the mean and the variance is stable. When there is proportionality between $\mu$ and $\sigma$, monitoring the coefficient of variation $\mathrm{CV}$ is suggested: the coefficient of variation is defined as the ratio between the standard deviation and the mean. Monitoring the $\mathrm{CV}$ is of interest in those processes where the mean and the standard deviation may vary but their ratio is expected to be unchanged. Since the CV is a relative measure of dispersion, a control chart for the CV allows to detect the unexpected change in the ratio between $\mu$ and $\sigma$. Several control charts using different methods such as Run-sum [1], CUSUM [2], synthetic [3,4], EWMA [5,6] have been discussed in literature to monitor the coefficient of variation of a normal distribution [7-10].

In the past, attention to the need of monitoring the Multivariate CV (MCV) has been invoked in [11]. Today, with the huge increase of data availability from industrial processes, there is an outstanding interest on tools for online monitoring of multivariate 
data. Therefore, considering the surveillance of the multivariate coefficient of variation is an issue worth of interest in SPM research. The first control chart aiming at monitoring the MCV was introduced by [12]: it is well known that Shewhart type control charts are easy to design and to interpret. Nevertheless, they are rather slow in the detection of small or moderate process shifts. For this reason several methods/strategies have been proposed in the SPC literature to overcome this weakness. For instance, the Run Sum control chart for the MCV was recently developed in [13]. In this paper, we propose a different approach for monitoring the MCV by using synthetic control charts.

Synthetic control charts have been widely used in literature to detect shifts in a process. The first introduction of the Synthetic $\bar{X}$ chart to the field of SPC was in [14]; then, its properties and design strategies have been thoroughly investigated by many authors [15-21] for several monitoring statistics. Recently, [22] investigated the effect of estimated process parameters on the performance of the Synthetic chart using a Markov chain model. They have shown that the run length (RL) performance of the Synthetic chart is quite different in the known and in the estimated process parameters cases. However, as far as we know, the Synthetic control chart for monitoring the MCV has never been considered in the SPC literature. Therefore, the goal of this paper is to present and investigate the performance of two one-sided Synthetic MCV control charts. The decision to implement two one-sided synthetic MCV control charts instead of a single two-sided synthetic MCV control chart is motivated by the following reasons:

- The sample MCV distribution is asymmetrical: therefore, designing different control limits allows to get equal values of the in-control $A R L$ for both the one-sided synthetic MCV control charts;

- there is more flexibility in the design of each one-sided synthetic MCV control chart: for example, if quality practitioners know that one direction of the outof-control condition can occur more frequently than another, the control limit of each one-sided synthetic MCV control chart can be properly tuned to have a higher sensitivity vs. the most frequent shift direction.

The rest of the paper is organised as follows. A brief review of the distribution of the sample multivariate coefficient of variation is given in Section 2. The implementation of the two one-sided synthetic MCV control charts is described in Section 3. Section 4 discusses the performance of the synthetic MCV control charts. In Section 5, we illustrate a real-world example using our design. The concluding remarks are given in Section 6 .

\section{A brief review of the distribution of the sample multivariate coefficient of variation}

In this Section, a brief overview of the distribution of the sample multivariate coefficient of variation is presented. Let us consider a random sample of size $n$, that is, $\mathbf{X}_{1}, \mathbf{X}_{2}, \ldots, \mathbf{X}_{n}$ from a $p$-variate normal distribution with mean vector $\boldsymbol{\mu}$ and covariance matrix $\boldsymbol{\Sigma}$, i.e., $\mathbf{X}_{i}=\left(x_{i, 1}, x_{i, 2}, \ldots, x_{i, p}\right) \sim N(\boldsymbol{\mu}, \boldsymbol{\Sigma}), i=1, \ldots, n$. According to [23], the MCV is defined as

$$
\gamma=\left(\boldsymbol{\mu}^{\top} \boldsymbol{\Sigma}^{-1} \boldsymbol{\mu}\right)^{-\frac{1}{2}}
$$

This definition is also used in [12] and [13]. Based on this definition of the MCV, 
we can introduce the sample MCV and discuss its distribution. Let $\overline{\mathbf{X}}$ and $\mathbf{S}$ be the sample mean vector and the sample variance-covariance matrix of $\mathbf{X}_{1}, \mathbf{X}_{2}, \ldots, \mathbf{X}_{n}$, i.e.,

$$
\overline{\mathbf{X}}=\frac{1}{n} \sum_{i=1}^{n} \mathbf{X}_{i}
$$

and

$$
\mathbf{S}=\frac{1}{n-1} \sum_{i=1}^{n}\left(\mathbf{X}_{i}-\overline{\mathbf{X}}\right)\left(\mathbf{X}_{i}-\overline{\mathbf{X}}\right)^{\top}
$$

Then the sample multivariate coefficient of variation $\hat{\gamma}$ is defined in [12]

$$
\hat{\gamma}=\left(\overline{\mathbf{X}}^{\top} \mathbf{S}^{-1} \overline{\mathbf{X}}\right)^{-\frac{1}{2}}
$$

In [12], the cumulative distribution function (cdf) of $\hat{\gamma}$ is computed as

$$
F_{\hat{\gamma}}(x \mid n, p, \gamma)=1-F_{F}\left(\frac{n(n-p)}{(n-1) p x^{2}} \mid p, n-p, \frac{n}{\gamma^{2}}\right),
$$

where $F_{F}\left(. \mid p, n-p, \frac{n}{\gamma^{2}}\right)$ is the non-central $F$ cdf with $p$ and $n-p$ degrees of freedom and non-centrality parameter $\frac{n}{\gamma^{2}}=n \boldsymbol{\mu}^{\top} \boldsymbol{\Sigma}^{-1} \boldsymbol{\mu}$. The probability density function (pdf) of $\hat{\gamma}$ can be easily obtained as

$$
f_{\hat{\gamma}}(x \mid n, p, \gamma)=\frac{2 n(n-p)}{(n-1) p x^{3}} f_{F}\left(\frac{n(n-p)}{(n-1) p x^{2}} \mid p, n-p, \frac{n}{\gamma^{2}}\right)
$$

where $f_{F}\left(. \mid p, n-p, \frac{n}{\gamma^{2}}\right)$ is the pdf of non-central $F$ distribution. In Figure 1, the pdf of $\hat{\gamma}$ is illustrated with $\gamma=0.3, p=\{2,3,4\}$ and $n=\{5,10,15\}$. This example demonstrates the asymmetry of the $\hat{\gamma}$ distribution. In a similar way, [12] presented the inverse distribution function (idf) of $\hat{\gamma}$ as follows

$$
F_{\hat{\gamma}}^{-1}(\alpha \mid n, p, \gamma)=\sqrt{\frac{n(n-p)}{(n-1) p}\left(\frac{1}{F_{F}^{-1}\left(1-\alpha \mid p, n-p, \frac{n}{\gamma^{2}}\right)}\right)},
$$

where $F_{F}^{-1}\left(. \mid p, n-p, \frac{n}{\gamma^{2}}\right)$ is the inverse cdf of the non-central $F$ distribution with $p$ and $n-p$ degrees of freedom and non-centrality parameter $\frac{n}{\gamma^{2}}$.

\section{Design and implementation of two one-sided the synthetic MCV control charts}

In general, a synthetic control chart consists of two sub-charts: a Shewhart sub-chart and a conforming run length $(C R L)$ sub-chart. The $C R L$ is defined as the number of inspected samples between two consecutive nonconforming samples, inclusive of the nonconforming sample at the end [24]. Figure 2 illustrates how the $C R L$ values are determined: in this example, we have $C R L_{1}=4, C R L_{2}=5$, and $C R L_{3}=3$. For the 


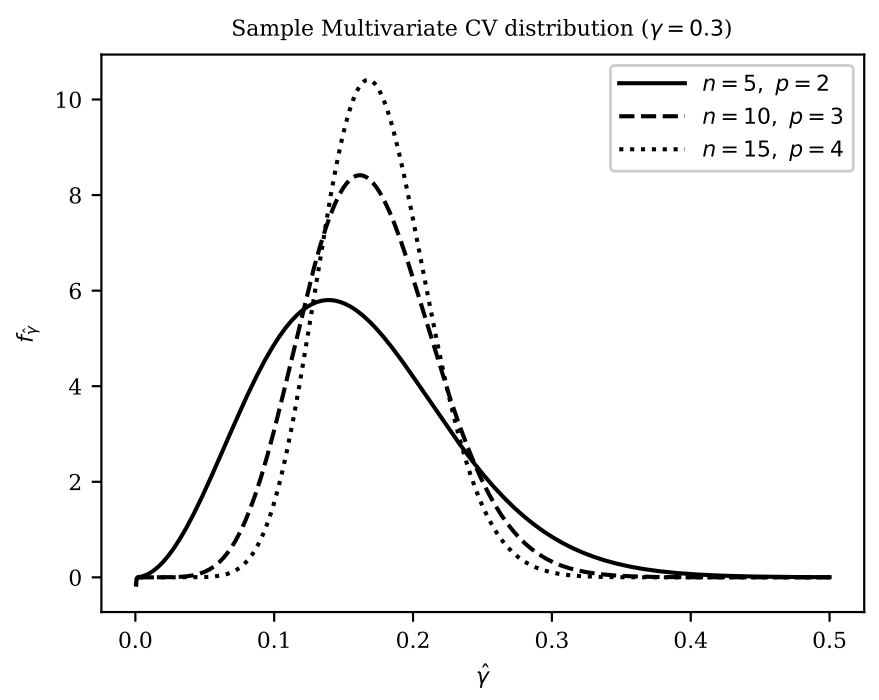

Figure 1. The pdf of $\hat{\gamma}$ for different values of parameters.

one-sided synthetic MCV control chart, the Shewhart sub-chart is defined as in [12]. Therefore, a $j$-th sample is declared as nonconforming if $\hat{\gamma}_{j}, j=1,2, \ldots$, falls beyond the selected control limits of the one-sided Shewhart MCV sub-chart. As mentioned, in this study, two one-sided synthetic control charts for monitoring the MCV are designed: a lower-sided synthetic MCV control chart and an upper-sided synthetic MCV control chart. For the purpose of distinguishing the two control charts, the superscript "-" will be used for the control limits of sub-charts running the lower-sided control chart, while the superscript " +" will be used for the control limits of sub-charts running the upper-sided control chart. The operation of the one-sided synthetic MCV control charts can be summarized as follows:

Step 1 Fix the sample size $n$. For the synthetic lower-sided (upper-sided) MCV chart, determine the control limit $H^{-}\left(H^{+}\right)$of the $C R L$ sub-chart and the lower (upper) control limit $L C L^{-}\left(U C L^{+}\right)$of the Shewhart MCV sub-chart. The synthetic lower-sided (upper-sided) MCV chart is run to detect a decreasing (increasing ) shift in $\hat{\gamma}_{j}$ with a single Lower (Upper) Control Limit $L C L^{-}\left(U C L^{+}\right)$, i.e. $U C L^{-}=+\infty\left(L C L^{+}=0\right)$.

Without any misunderstanding, the lower control limit $L C L^{-}$of the one-sided lower Shewhart MCV sub-chart and the upper control limit $U C L^{+}$of the onesided upper Shewhart MCV sub-chart can be simply written as $L C L$ and $U C L$, respectively.

Step 2 At each sampling point $j=1,2, \ldots$, take a sample of size $n$ from the process and evaluate the sample MCV $\hat{\gamma}_{j}$ as in (2).

Step 3 If $\hat{\gamma}_{j}>L C L$ (respectively, $\hat{\gamma}_{j}<U C L$ in case of the upper-sided synthetic $\mathrm{MCV}$ control chart), this sample is considered as conforming in the $C R L$ subchart: the process is considered to be in-control and the control flow goes back to step 2 to take the next sample. Otherwise, the sample is nonconforming and the control flow goes to the next step.

Step 4 If $C R L>H^{-}$(respectively, $C R L>H^{+}$in case of the upper-sided one-sided lower Shewhart MCV sub-chart) the process is deemed to be in-control and the 


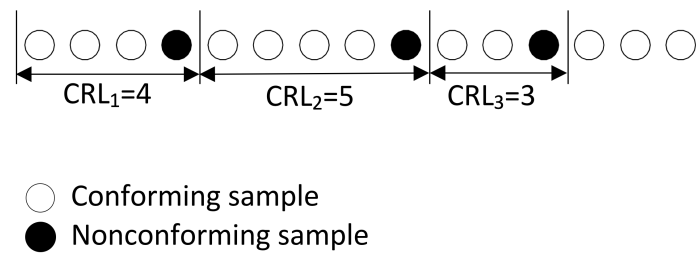

Figure 2. The conforming run length (CRL).

control flow moves back to step 2. Otherwise, the process is declared to be in the out-of-control state and the control flow advances to the next step.

Step 5 Signal an out-of-control status to indicate a process shift. Find and remove potential assignable cause(s). Then move back to Step 2 .

We assume that the occurrence of an out-of-control condition shifts the in-control MCV, denoted by $\gamma_{0}$, to the out-of-control MCV, defined as $\gamma_{1}=\tau \gamma_{0}$, where $\tau>0$ is the shift size. Values of $\tau \in(0,1)$ correspond to a decrease of the in-control MCV $\gamma_{0}$, while values of $\tau>1$ correspond to an increase of the in-control MCV $\gamma_{0}$. In order to obtain the run length properties of the lower-sided Synthetic MCV control chart, similarly to [15], we use a Markov chain where the $\left(H^{-}+2, H^{-}+2\right)$ transition probability matrix $\mathbf{P}$ is equal to

$$
\mathbf{P}=\left(\begin{array}{cc}
\mathbf{Q} & \mathbf{r} \\
\mathbf{0}^{\top} & 1
\end{array}\right)=\left(\begin{array}{cccccc|c}
1-\theta & \theta & 0 & \cdots & \cdots & 0 & 0 \\
0 & 0 & 1-\theta & \ddots & & 0 & \theta \\
\vdots & & \ddots & \ddots & \ddots & \vdots & \vdots \\
\vdots & & & \ddots & 1-\theta & 0 & \vdots \\
0 & \cdots & \cdots & \cdots & 0 & 1-\theta & \theta \\
1-\theta & 0 & \cdots & \cdots & \cdots & 0 & \theta \\
\hline 0 & \cdots & \cdots & \cdots & \cdots & 0 & 1
\end{array}\right),
$$

where $\mathbf{0}^{\boldsymbol{\top}}=(0,0, \ldots, 0)$ is a $\left(1, H^{-}+1\right)$ row vector, $\mathbf{Q}$ is a $\left(H^{-}+1, H^{-}+1\right)$ transition probability matrix for the transient states, the $\left(H^{-}+1,1\right)$ column vector $\mathbf{r}$ satisfies $\mathbf{r}=\mathbf{1}-\mathbf{Q} \mathbf{1}$ with $\mathbf{1}=(1,1, \ldots, 1)^{\boldsymbol{\top}}$ and $\theta=P\left(\gamma_{j} \leq L C L\right)$ is the probability of a nonconforming sample on the MCV sub-chart. In case of the upper-sided synthetic MCV control chart, $H^{-}$is replaced by $H^{+}$and $\theta=P\left(\gamma_{j} \geq U C L\right)$.

- For the lower-sided control chart

$$
\theta=F_{\hat{\gamma}}\left(L C L \mid n, p, \gamma_{1}\right) .
$$

- For the upper-sided control chart

$$
\theta=1-F_{\hat{\gamma}}\left(U C L \mid n, p, \gamma_{1}\right),
$$

Since the calculation in case of the lower-sided control chart is similar to the one of an upper-sided control chart, in the next discussion we only focus on the synthetic lowersided MCV control chart. The differences with the upper-sided control chart will be discussed when it is required. The corresponding $\left(H^{-}+1,1\right)$ vector $\mathbf{q}$ of the initial prob- 
abilities associated with the $H^{-}+1$ transient states is equal to $\mathbf{q}=\left(q_{1}, \ldots, q_{H^{-}+1}\right)^{\top}$. As proposed by [25] and [26], the mean of the run length $(A R L)$ and the standarddeviation $(S D R L)$ of the run length of the synthetic MCV control chart are computed as

$$
\begin{aligned}
A R L_{\mathrm{ZS}} & =\nu_{1}, \\
S D R L_{\mathrm{ZS}} & =\sqrt{\nu_{2}-\nu_{1}^{2}+\nu_{1}},
\end{aligned}
$$

with

$$
\begin{aligned}
& \nu_{1}=\mathbf{q}^{\boldsymbol{\top}}(\mathbf{I}-\mathbf{Q})^{-1} \mathbf{1}, \\
& \nu_{2}=2 \mathbf{q}^{\boldsymbol{\top}}(\mathbf{I}-\mathbf{Q})^{-2} \mathbf{Q} \mathbf{1},
\end{aligned}
$$

and $\mathbf{q}=(0,1,0, \ldots, 0)^{\top}$, i.e. the initial state is the second one, as suggested in [22]. With this vector $q$ of initial probabilities, we are able to obtain the zero-state performance of the synthetic MCV control chart. The subscript "ZS" stands for zero-state condition.

It is important to note that, if the process is running for some time in the incontrol condition, it will reach quite rapidly the steady-state mode. In order to study the long term properties of the synthetic MCV control chart, it is also appropriate to investigate the steady-state $A R L$. Using the Markov Chain approach, the cyclical steady-state mean $\left(A R L_{\mathrm{SS}}\right)$ and the standard-deviation $\left(S D R L_{\mathrm{SS}}\right)$ of the run length of the Synthetic MCV control chart are found as follows

$$
\begin{aligned}
A R L_{\mathrm{SS}} & =\nu_{s 1} \\
S D R L_{\mathrm{SS}} & =\sqrt{\nu_{s 2}-\nu_{s 1}^{2}+\nu_{s 1}}
\end{aligned}
$$

with

$$
\begin{aligned}
& \nu_{s 1}=\boldsymbol{\psi}^{\boldsymbol{\top}}(\mathbf{I}-\mathbf{Q})^{-1} \mathbf{1}, \\
& \nu_{s 2}=2 \boldsymbol{\psi}^{\boldsymbol{\top}}(\mathbf{I}-\mathbf{Q})^{-2} \mathbf{Q} \mathbf{1}
\end{aligned}
$$

where the vector $\boldsymbol{\psi}$ is the cyclical steady state distribution. Following [27], we conclude that the cyclical steady-state vector is given by $\boldsymbol{\psi}=\frac{\left(\mathbf{I}-\mathbf{Q}^{\top}\right)^{-1} \mathbf{q}}{\mathbf{1}^{\boldsymbol{\top}}\left(\mathbf{I}-\mathbf{Q}^{\top}\right)^{-1} \mathbf{q}}$, where $\mathbf{q}$ is the $\left(H^{-}+1,1\right)$ vector, $\mathbf{q}=(0,1,0, \ldots, 0)^{\top}$.

The statistical design of the synthetic lower-sided MCV control control chart is a nonlinear optimization problem aimed at selecting the optimal parameters $H^{-*}$ such that

$$
\left(H^{-*}, L C L^{*}\right)=\underset{\left(H^{-}, L C L\right)}{\arg \min } A R L\left(n, p, H^{-}, L C L, \gamma_{0}, \tau\right),
$$

subject to

$$
A R L\left(n, p, H^{-}, L C L, \gamma_{0}, \tau=1\right)=A R L_{0},
$$

where $A R L\left(n, p, H^{-}, L C L, \gamma_{0}, \tau\right)$ is either the zero-state $A R L_{\mathrm{ZS}}$ or the cyclical steady state $A R L_{\mathrm{SS}}$ of the synthetic lower-sided MCV control chart; $A R L_{0}$ is the nominal 
"in-control" (zero-state or cyclical steady state) $A R L$. The optimization procedure can be summarized as follows:

Step 1 Set $n, p, \gamma_{0}, \tau$ and $A R L_{0}$. Set $A R L_{o p t}=+\infty$;

Step 2 Initialize $H^{-}=1$;

Step 3 Compute $L C L$ through constraint (16);

Step 4 Compute $A R L$ from the current design solution $H^{-}$by using either (7) or (11);

Step 5 If $A R L<A R L_{\text {opt }}$, then $A R L_{\text {opt }}=A R L$ and $H^{-*}=H^{-}, L C L^{*}=L C L$. Set $H^{-}=H^{-}+1$ and go back to Step 3. Otherwise, go to Step 6;

Step 6 Take the current solution $\left(H^{-*}, L C L^{*}\right)$ as the optimal set of design parameters for the synthetic MCV control chart.

Similar to [2], the optimal $L C L$ is found numerically by means of a non-linear equation solver developed in the Matlab software environment. For the case of an upper-sided control chart, $H^{-}$is replaced by $H^{+}$and $L C L$ by $U C L$. Similarly, the optimization problem is as follows:

$$
\left(H^{+*}, U C L^{*}\right)=\underset{\left(H^{+}, U C L\right)}{\arg \min } A R L\left(n, p, H^{+}, U C L, \gamma_{0}, \tau\right)
$$

subject to

$$
A R L\left(n, p, H^{+}, U C L, \gamma_{0}, \tau=1\right)=A R L_{0},
$$

\section{Statistical Performance Study}

\subsection{Performance with known shift size}

In this Section, we will use the $A R L, S D R L$ to evaluate the performance of the synthetic MCV charts. When the process is in-control, the target $A R L$ is denoted by $A R L_{0}$ : here, we set $A R L_{0}=370.4$. For the lower side synthetic MCV control chart, the optimal design parameters $\left(H^{-*}, L C L^{*}\right)$ and zero-state $A R L$ are shown in Table 2 for different combinations of $n=\{5,10,15\}, p=\{2,3,4\}, \gamma_{0}=\{0.1,0.2,0.3,0.4,0.5\}$ and $\tau=\{0.5,0.75,0.9\}$. Table 3 illustrates the optimal design parameters $\left(H^{+*}, U C L^{*}\right)$ and the zero-state the $A R L$ for the upper-sided synthetic MCV control chart with the same combinations of $n, p, \gamma_{0}$ as in Table 2 but $\tau=\{1.1,1.25,1.5\}$. In case of steady-state condition, the same results are presented in Table 4 and Table 5.

From Table 2-5, the $H^{-*}, H^{+*}, A R L$ and $S D R L$ values for both the lower-sided and the upper-sided control charts are larger when $\tau$ is close to 1 . For example, by referring to Table 2 under the zero-state condition, with $p=2, n=10, \gamma_{0}=0.1$ and $\tau=0.5$, the values of $A R L_{\mathrm{ZS}}$ and $S D R L_{\mathrm{ZS}}$ are 1.5 and 1.1, respectively; while $A R L_{\mathrm{ZS}}$ and $S D R L_{\mathrm{ZS}}$ are 105.4 and 128.2 , respectively when $\tau=0.9$. This was expected, since the shift is smaller when $\tau$ is close to 1 . Similar behavior is observed with regards to the sample size $n$ : the larger is the sample size, the smaller is the number of samples needed to detect an out-of-control situation in average; also the $S D R L$ is smaller. For instance, as shown in Table 4 under the steady-state condition with $\gamma_{0}=0.1, \tau=0.75, p=4$; the $A R L_{\mathrm{SS}}$ and $S D R L_{\mathrm{SS}}$ values are 212.5 and 211.8 , respectively when $n=5$, with $n=10$, these values are $A R L_{\mathrm{SS}}=32.9$ and $S D R L_{\mathrm{SS}}=31.7$, respectively. This improved performance with $n$ is more significant for larger values of $p$. Therefore, a larger sample size is recommended when more variables are monitored. 
It is also observed that the $A R L$ and $S D R L$ values are larger for the lower-sided control chart compared to the upper-sided control chart. On the contrary, the $H^{-*}$ values for the lower-sided control chart are smaller than the $H^{+*}$ values for the uppersided control chart. For example, in Tables 2-3, with $p=2, n=10$ and $\gamma_{0}=0.1$, $A R L_{\mathrm{ZS}}, S D R L_{\mathrm{ZS}}$ and $H^{-*}$ of the lower-sided control chart are 105.4, 128.2 and 11, respectively, when $\tau=0.9$, while $A R L_{\mathrm{ZS}}, S D R L_{\mathrm{ZS}}$ and $H^{+*}$ of the upper-sided control chart are 44.1, 57.4 and 31, respectively, when $\tau=1.1$. In the other words, it is easier to detect the upward shift in MCV than the downward shift. The different behavior between the lower-sided control chart and the upper-sided control chart is due to the asymmetric of the distribution of the MCV, see Figure 1. In practice, the detection of an upward shift is usually more important, as an upward shift indicates an increase of relative variation with regard to the mean.

Both the optimal values $H^{+*}$ and $H^{-*}$ are smaller when the shift is large. In addition, when the sample size $n$ increases, we observed that, the values of $H^{+*}$ decreases in both zero-state and steady-state conditions. On the other hand, the values of $H^{-*}$ increase in both conditions when $n$ increases. The design optimization algorithm runs to minimize the average run length: due to the asymmetry of the distribution of the MCV, the trends of $H^{+*}$ and $H^{-*}$ are different. Moreover, it is observed that, in some cases, the values $H$ are larger than the $A R L$ values. For instance, at the end of Table 3 , with $p=4, n=5, \tau=1.5$, the values of $H^{+*}$ and $A R L_{Z S}$ are 26 and 19.5, respectively. In fact, as illustrated in Figure 3, the decrease of $A R L$ with respect to $H$ is insignificant when the $A R L$ is close to the minimum.

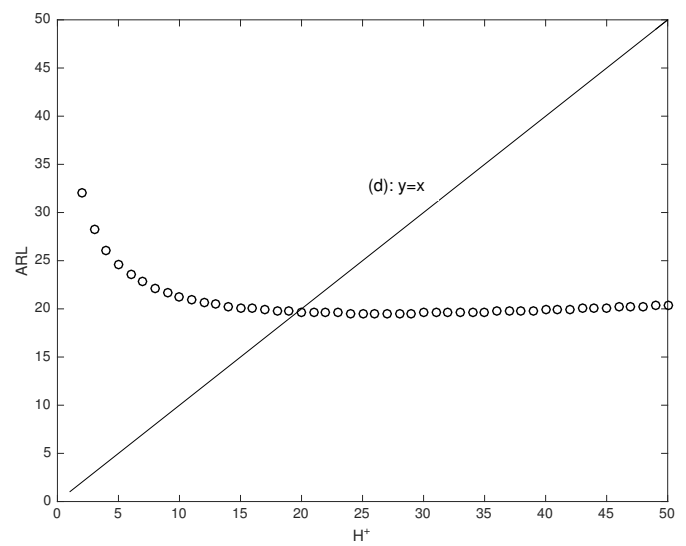

Figure 3. The variation of $A R L$ with respect to $H$.

For a larger value of $\gamma_{0}$, a larger $L C L^{*}$ for the lower-sided control chart is adopted; the value of $U C L^{*}$ is also larger for the upper side chart. When the sample size $n$ increases, $L C L^{*}$ also increases for the lower side control chart, while $U C L^{*}$ does not significantly change. It is also observed that the higher in-control MCV $\gamma_{0}$ slightly increases the average number of samples needed to detect a process shift.

\section{PLEASE INSERT TABLES 2, 3, 4, 5 HERE}

The performance comparison between the $A R L$ values of the one-sided Synthetic MCV control charts and the $A R L$ values of the one-sided Shewhart MCV control charts proposed in [12] is provided in Table 6 . The The performance comparison has 
been undertaken by defining the following index $\Delta_{E}$

$$
\Delta_{E}=\frac{A R L_{\mathrm{SH}}-A R L_{\text {syn }}}{A R L_{\mathrm{SH}}} \times 100,
$$

where $A R L_{\mathrm{SH}}$ is the average run length value for the Shewhart MCV control chart, while $A R L_{\text {syn }}$ is the average run length value for the synthetic MCV control chart. If $\Delta_{E}>0$, then the Synthetic MCV charts outperform the Shewhart MCV charts; if $\Delta_{E}<0$, then the Shewhart MCV charts outperform the Synthetic MCV charts. The obtained results presented in Tables 6 , rounded to the nearest integer, show that the Synthetic MCV charts outperform the Shewhart MCV charts for both the lower-sided and the upper-sided control charts. The Synthetic MCV charts are significantly better than the Shewhart MCV charts when the shift size is large, i.e. $|\tau-1|$ is large.

\section{PLEASE INSERT TABLE 6 HERE}

\subsection{Performance with unknown shift size}

We already know that the synthetic MCV control charts can be optimally designed in terms of $A R L$ for quickly detecting anticipated shift sizes. However, in practice, the shift size $\tau$ to the out-of-control condition cannot be predicted with sufficient precision. In order to overcome this problem, the uncertainty related to the shift size prediction can be tackled by considering $\tau$ as a random variable and selecting a statistical distribution to model it. Several potential statistical distributions have been considered in the literature, see [28]. If the quality practitioner wants to get an optimal design of a control chart with respect to a range of shifts sizes $\Omega=[a, b]$, without any preference for a specific size, then the uniform distribution can be selected to give an equal weight to each shift size included within the interval $\Omega$, see [28]. Therefore, in the second step of our numerical analysis, we have computed new optimal couples $\left(H^{-*}, L C L^{*}\right)$ or $\left(H^{+*}, U C L^{*}\right)$, such that

- For the lower-sided control chart:

$$
\left(H^{-*}, L C L^{*}\right)=\underset{\left(H^{-}, L C L\right)}{\arg \min } \operatorname{EARL}\left(n, p, H^{-}, L C L, \gamma_{0}, \tau\right)
$$

subject to the constraint

$$
\begin{aligned}
& \operatorname{EARL}\left(n, p, H^{-}, U C L^{-}, \gamma_{0}, \tau=1\right) \\
& =A R L\left(n, p, H^{-}, U C L^{-}, \gamma_{0}, \tau=1\right)=A R L_{0},
\end{aligned}
$$

- For the upper-sided control chart:

$$
\left(H^{+*}, U C L^{*}\right)=\underset{\left(H^{+}, U C L\right)}{\arg \min } \operatorname{EARL}\left(n, p, H^{+}, U C L, \gamma_{0}, \tau\right)
$$

subject to the constraint

$$
\begin{aligned}
& \operatorname{EARL}\left(n, p, H^{+}, U C L, \gamma_{0}, \tau=1\right) \\
& =A R L\left(n, p, H^{+}, U C L, \gamma_{0}, \tau=1\right)=A R L_{0},
\end{aligned}
$$


where $E A R L$ (Expected Average Run Length) is equal to

$$
E A R L=\int_{\Omega} A R L \times f_{\tau}(\tau) d \tau,
$$

with $f_{\tau}(\tau)=\frac{1}{b-a}$ for $\tau \in \Omega=[a, b]$ and $A R L$ is defined as in (7). The new optimal couples $\left(\left(H^{-*}, L C L^{*}\right)\right.$ and $\left.\left(H^{+*}, U C L^{*}\right)\right)$ and the values of $E A R L$ are presented in Tables $7-8$ for $\Omega=[0.5,1$ ) (decreasing case, denoted by $(\mathrm{D})$ ) and $\Omega=(1,2]$ (increasing case, denoted by (I)). Table 7 presents the zero-state performance, while Table 8 presents the steady state performance.

Similar to the known shift case, EARL values are larger for the lower-sided chart, if compared to the upper-sided chart. While the $H^{-*}$ values are small compared to the $H^{+*}$ values. Detecting the upward shift in MCV is easier than the downward shift. As expected, the control chart sensitivity improves with the sample size. Increasing the sample size $n$ also leads to a tighter in-control interval on the chart. It is also observed that the higher in-control MCV $\gamma_{0}$ slightly increases the EARL values. With regards to the number of monitored variables, the values of $E A R L$ increase with $p$. Therefore, a larger sample size is always recommended when we have more correlated variables.

\section{PLEASE INSERT TABLE 7, 8 HERE}

The performance comparison between the $A R L$ values of the Synthetic MCV control charts with the $A R L$ values for the Shewhart MCV control charts (on both uppersided and lower-sided cases) is also conducted. The comparison indices computed by (19) are provided in Table 9. The results show that the Synthetic MCV charts always outperform the Shewhart MCV chart in the unknown shift case. Moreover, the Synthetic MCV control charts perform much better when more samples are taken.

\section{PLEASE INSERT TABLE 9 HERE}

In addition, we also make a direct comparison of $A R L$ values with the run sum MCV control chart developed by [13]. The experiments in [13] are only conducted with $\gamma_{0}=\{0.1,0.3,0.5\}, p=\{2,3\}$ and $n=\{5,10\}$, see Tables $1-4$ in [13]. The value of $A R L_{0}$ in this comparison is 370 . Under the zero-state condition, as shown in Table 10, the upper-sided synthetic control chart is able to detect sooner the upward shift in MCV. For example, with $\gamma_{0}=0.1, \tau=1.25, p=3$ and $n=10$, the results of $A R L$ are 9.1 and 11.8 for the synthetic upper-sided MCV and run sum MCV control charts, respectively. However, the run sum MCV control chart performs better than the synthetic lower-sided MCV control chart in detecting the downward shift. Similarly, when the shift size is unknown, the synthetic MCV control charts are faster than the run sum MCV control chart in detecting upward shifts.

\section{PLEASE INSERT TABLE 10 HERE}

Finally, we compare the performance of the synthetic MCV control charts with the very recent adaptive MCV control charts designed in [29]. In order to make a fair comparison, only the upward control charts are investigated and the parameters used in [29] are $\gamma_{0}=\{0.1,0.3,0.5\}, p=\{2,3\}, \tau=\{1.25,1.5\}$ and $n=\{5,10\}$, see Tables 3-4 in [29]. And a Synthetic chart is considered as a fixed sampling interval control chart with $h=1$. As presented in Table 11, the variable sample size and sampling interval (VSSI) and variable sample size (VSS) MCV control charts outperform the Synthetic MCV control chart. On the other hand, the Synthetic MCV control chart gives a better performance than the variable sampling interval (VSI) control chart 
in most cases. However, it should be noted that, in [29], the results are only given under the steady-state condition. Moreover, the Synthetic MCV charts work well under the zero-state condition. Therefore, we cannot draw a general conclusions about the performance difference.

\section{PLEASE INSERT TABLE 11 HERE}

\section{Illustrative example}

In this Section, we discuss the implementation of the upper-sided and the lower-sided control charts. The context of the example presented here is similar to the one introduced in [13]. As discussed in [13], the data are obtained from a company, for which the quality characteristics are the inner diameters A and B, whose measurements are presented by $\left(X_{1}\right)$ and $\left(X_{2}\right)$. From phase I, the value of MCV has been estimated, i.e. $\hat{\gamma}_{0}=0.089115$. The data collected during the phase II process with sample size $n=5$ are shown in Table 1.

\begin{tabular}{ccccccc}
\hline Sample number $i$ & $X_{1, i}$ & $X_{2, i}$ & $S_{1, i}^{2}$ & $S_{2, i}^{2}$ & $S_{12, i}$ & $\hat{\gamma}_{i}$ \\
\hline 1 & 7.781 & 1.592 & 1.164 & 0.734 & 0.35645 & 0.113710 \\
2 & 7.385 & 1.804 & 1.006 & 1.667 & 0.96049 & 0.104890 \\
3 & 7.988 & 2.260 & 0.762 & 0.359 & 0.17373 & 0.108870 \\
4 & 8.189 & 2.100 & 1.885 & 0.470 & 0.13026 & 0.156790 \\
5 & 7.436 & 2.061 & 1.404 & 0.519 & 0.08280 & 0.139290 \\
6 & 6.746 & 2.289 & 0.846 & 0.811 & 0.43835 & 0.133240 \\
7 & 7.356 & 1.917 & 0.197 & 2.587 & 0.01597 & 0.059996 \\
8 & 8.492 & 1.845 & 1.460 & 1.746 & 1.42051 & 0.055093 \\
9 & 7.272 & 1.580 & 1.353 & 0.345 & 0.27988 & 0.117710 \\
10 & 7.585 & 1.568 & 1.098 & 0.788 & 0.41252 & 0.109610 \\
11 & 7.734 & 1.709 & 0.952 & 0.228 & 0.11462 & 0.102440 \\
12 & 8.160 & 1.498 & 1.598 & 1.178 & 1.00757 & 0.122950 \\
13 & 7.102 & 2.661 & 1.508 & 0.945 & 0.73607 & 0.101260 \\
14 & 8.392 & 1.883 & 0.536 & 0.706 & 0.23234 & 0.085637 \\
15 & 7.592 & 2.531 & 0.256 & 0.563 & 0.24827 & 0.043489 \\
16 & 8.141 & 2.093 & 0.394 & 0.603 & 0.25584 & 0.072202 \\
17 & 7.883 & 2.490 & 1.321 & 1.179 & 0.65037 & 0.142430 \\
18 & 7.886 & 2.877 & 0.883 & 1.431 & 0.22524 & 0.106680 \\
19 & 7.830 & 1.008 & 0.878 & 0.558 & 0.14223 & 0.112090 \\
20 & 8.196 & 1.482 & 0.791 & 0.220 & 0.13724 & 0.088460 \\
\hline
\end{tabular}

Table 1. Illustrative example of Phase II dataset.

In this case, the upper-sided and the lower-sided control charts, which are designed for a quick detection of a $25 \%$ increasing shift (i.e., $\tau=1.25$ ) and a $25 \%$ decreasing shift (i.e., $\tau=0.75)$ in the MCV, are implemented simultaneously. Based on the optimization procedure in Sec. 3 with $A R L_{0}=370.4$, we obtain $\left(H^{+*}=22, U C L^{*}=\right.$ $0.1487)$ and $\left(H^{-*}=3, L C L^{*}=0.0221\right)$ for the upper-sided and the lower-sided control charts respectively.

The corresponding values $\hat{\gamma}_{i}$ are presented in the rightmost column of Table 1 and plotted in Figures 4 and 5 , respectively. At sample \#4, a point is plotted above $U C L^{*}=$ 0.1487 and a conforming run length $C R L_{1}=4<H^{+*}=22$ is recorded. Therefore, 


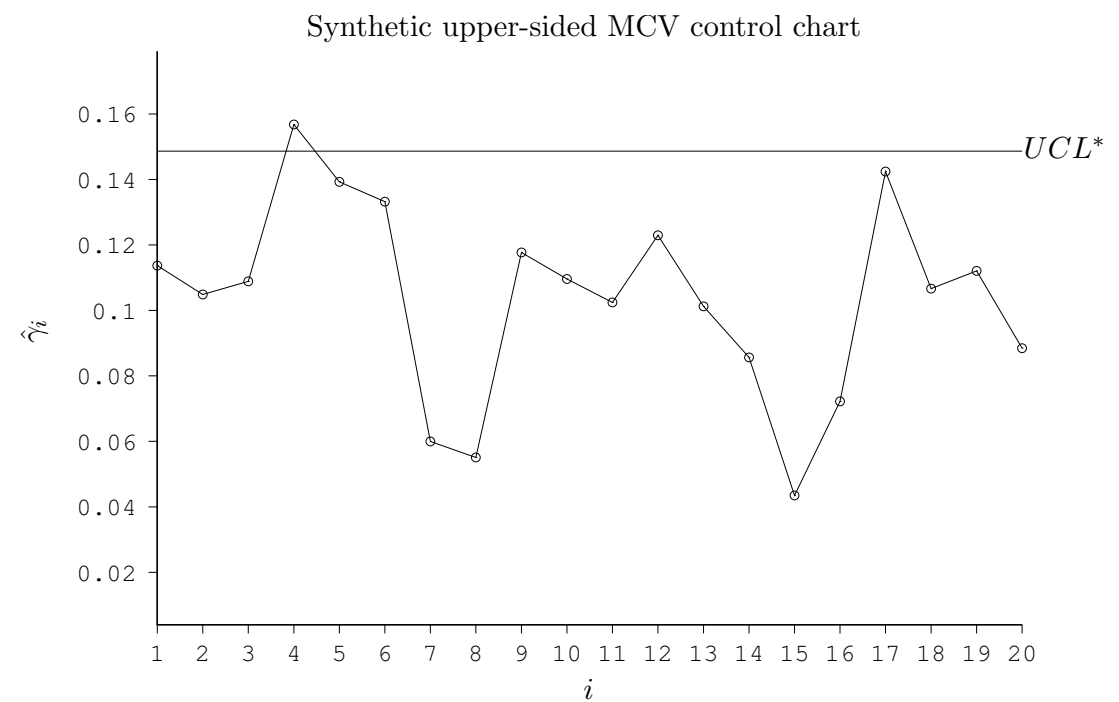

Figure 4. Synthetic upper-sided control chart corresponding to Phase II data set in Table 1.

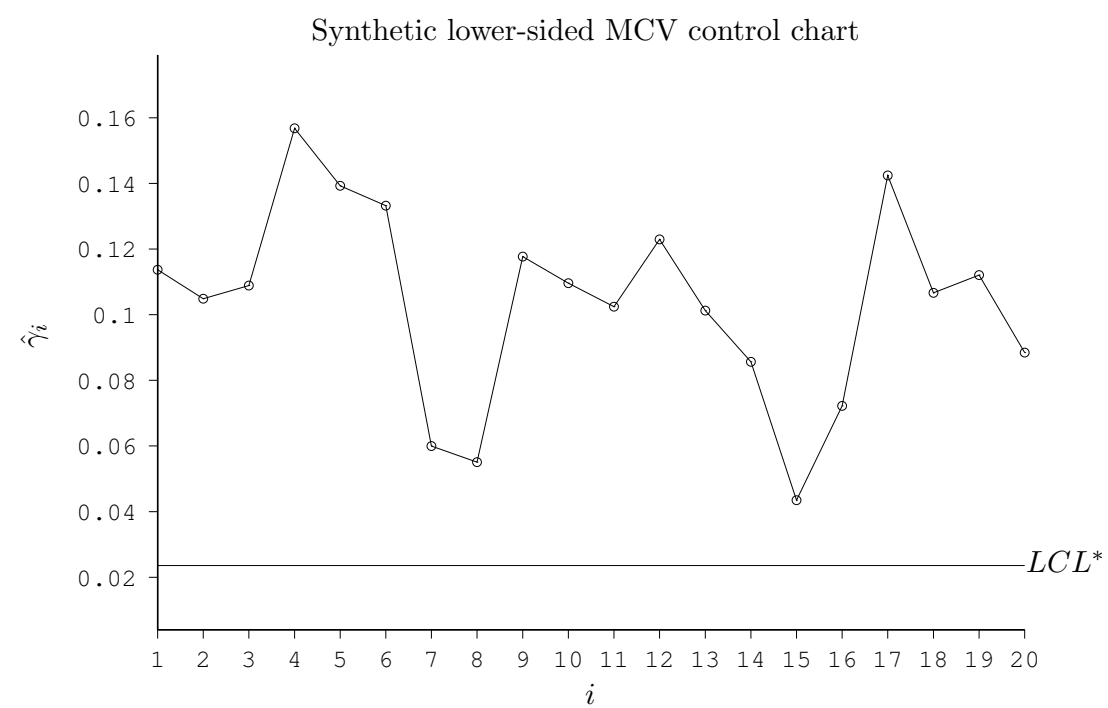

Figure 5. Synthetic lower-sided control chart corresponding to Phase II data set in Table 1. 
the upper-sided control chart triggers an alarm signaling at sample \#4. Conversely, the lower-sided control chart does not detect any out-of-control signal.

\section{Concluding remarks}

Monitoring the multivariate coefficient of variation is recently receiving growing attention in the context of SPC. In this study, we proposed two one-sided synthetic control charts for monitoring the MCV: synthetic lower-sided and synthetic uppersided MCV control charts. By combining the Shewhart chart with a conforming run length $(C R L)$ chart, the performance of the control charts are significantly improved. The paper presents both zero-state and steady-state conditions for the control charts. For both fixed values of the shift size $\tau$ and unknown shift size, several tables presenting the optimal design parameters and out-of-control $A R L$ corresponding to different values of the in-control MCV $\gamma_{0}$ have been discussed. In this paper we also illustrate a practical example from a manufacturing process.

The synthetic control charts for monitoring MCV are simple to implement, yet effectively enhance the detection ability. A comparison with the one-sided Shewhart MCV control charts demonstrated the clear outperformance of the synthetic one-sided MCV control charts. The synthetic upper-sided MCV chart also performs better the run sum MCV chart. The future research about monitoring the MCV can be extended to adaptive schemes with variable parameters and the design of EWMA and CUSUM control charts.

\section{References}

[1] Teoh W, Khoo M, Castagliola P, et al. Run-sum control charts for monitoring the coefficient of variation. European Journal of Operational Research. 2017;257(1):144-158.

[2] Tran P, Tran KP. The Efficiency of CUSUM schemes for monitoring the Coefficient of Variation. Applied Stochastic Models in Business and Industry. 2016;32(6):870-881.

[3] Calzada ME, Scariano SM. A synthetic control chart for the coefficient of variation. Journal of Statistical Computation and Simulation. 2013;83(5):853-867.

[4] Tran KP, Nguyen HD, Nguyen QT, et al. One-sided synthetic control charts for monitoring the coefficient of variation with measurement errors. In: 2018 IEEE International Conference on Industrial Engineering and Engineering Management (IEEM); Dec; 2018. p. $1667-1671$.

[5] Zhang J, Li Z, Chen B, et al. A new exponentially weighted moving average control chart for monitoring the coefficient of variation. Computers \& Industrial Engineering. 2014; 78:205-212.

[6] Castagliola P, Celano G, Psarakis S. Monitoring the coefficient of variation using EWMA charts. Journal of Quality Technology. 2011;43(3):249-265.

[7] Khaw KW, Khoo MB, Yeong WC, et al. Monitoring the coefficient of variation using a variable sample size and sampling interval control chart. Communications in StatisticsSimulation and Computation. 2017;46(7):5772-5794.

[8] Yeong WC, Khoo MB, Lim SL, et al. A direct procedure for monitoring the coefficient of variation using a variable sample size scheme. Communications in Statistics-Simulation and Computation. 2017;46(6):4210-4225.

[9] You HW, Khoo MB, Castagliola P, et al. Monitoring the coefficient of variation using the side sensitive group runs chart. Quality and Reliability Engineering International. 2016; 32(5):1913-1927. 
[10] Kang CW, Lee MS, Seong YJ, et al. A control chart for the coefficient of variation. Journal of quality technology. 2007;39(2):151-158.

[11] Aerts S, Haesbroeck G, Ruwet C. Multivariate coefficients of variation: Comparison and influence functions. Journal of Multivariate Analysis. 2015;142:183-198.

[12] Yeong W, Khoo MBC, LTeoh W, et al. A control chart for the multivariate coefficient of variation. Quality and Reliability Engineering International. 2016;32(3):1213-1225.

[13] Lim AJ, Khoo MB, Teoh W, et al. Run sum chart for monitoring multivariate coefficient of variation. Computers \& Industrial Engineering. 2017;109:84-95.

[14] Wu Z, Spedding T. A Synthetic Control Chart for Detecting Small Shifts in the Process Mean. Journal of Quality Technology. 2000;32(1):32-38.

[15] Davis RB, Woodall WH. Evaluating and improving the synthetic control chart. Journal of Quality Technology. 2002;34(2):200.

[16] Chen F, Huang H. A Synthetic Control Chart for Monitoring Process Dispersion with Sample Range. International Journal of Advanced Manufacturing Technology. 2005;26(78):842-851.

[17] Huang H, Chen F. A Synthetic Control Chart for Monitoring Process Dispersion with Sample Standard Deviation. Computers \& Industrial Engineering. 2005;49(2):221-240.

[18] Costa A, Rahim M. A Synthetic Control Chart for Monitoring the Process Mean and Variance. Journal of Quality in Maintenance Engineering. 2006;12(1):81-88.

[19] Costa A, de Magalhaes M, Epprecht E. Monitoring the Process Mean and Variance Using a Synthetic Chart with Two-stage Testing. International Journal of Production Research. 2009;47(18):5067-5086.

[20] Wu Z, Ou Y, Castagliola P, et al. A Combined Synthetic\&X Chart for Monitoring the Process Mean. International Journal of Production Research. 2010;48(24):7423-7436.

[21] Khoo M, Lee H, Wu Z, et al. A Synthetic Double Sampling Control Chart for the Process Mean. IIE Transactions. 2011;43(1):23-38.

[22] Zhang Y, Castagliola P, Wu Z, et al. The synthetic [xbar] chart with estimated parameters. IIE Transactions. 2011;43(9):676-687.

[23] Nikulin M, Voinov V. Unbiased estimators and their applications. In: International encyclopedia of statistical science. Springer; 2011. p. 1619-1621.

[24] You H, Khoo MB, Castagliola P, et al. Side sensitive group runs $\mathrm{x}^{-}$chart with estimated process parameters. Computational Statistics. 2015;30(4):1245-1278.

[25] Neuts M. Matrix-Geometric Solutions in Stochastic Models: an Algorithmic Approach. Baltimore, MD: Johns Hopkins University Press; 1981.

[26] Latouche G, Ramaswami V. Introduction to Matrix Analytic Methods in Stochastic Modelling. Philadelphia, PA: Series on Statistics and Applied Probability. SIAM; 1999.

[27] Darroch J, Seneta E. On quasi-stationary distributions in absorbing discrete-time finite markov chains. Journal of Applied Probability. 1965;2(1):88-100.

[28] Celano G, Castagliola P, Nenes G, et al. Performance of $t$ Control Charts in Short Runs with Unknown Shift Sizes. Computers \& Industrial Engineering. 2013;64:56-68.

[29] Khaw KW, Khoo MB, Castagliola P, et al. New adaptive control charts for monitoring the multivariate coefficient of variation. Computers \& Industrial Engineering. 2018;126:595610 . 


\begin{tabular}{|c|c|c|c|c|c|c|c|c|c|c|c|c|c|}
\hline \multirow{3}{*}{$p$} & \multirow{3}{*}{$\tau$} & \multicolumn{4}{|c|}{$n=5$} & \multicolumn{4}{|c|}{$n=10$} & \multicolumn{4}{|c|}{$n=15$} \\
\hline & & $H^{-*}$ & $L C L^{*}$ & $A R L_{\mathrm{ZS}}$ & $S D R L_{\mathrm{ZS}}$ & $H^{-*}$ & $L C L^{*}$ & $A R L_{\mathrm{ZS}}$ & $S D R L_{\mathrm{ZS}}$ & $H^{-*}$ & $L C L^{*}$ & $A R L_{\mathrm{ZS}}$ & $S D R L_{\mathrm{ZS}}$ \\
\hline & & & & & & \multicolumn{4}{|c|}{$\gamma_{0}=0.1$} & & & & \\
\hline 2 & 0.50 & 2 & 0.0266 & 10.6 & 12.8 & 2 & 0.0523 & 1.5 & 1.1 & 2 & 0.0624 & 1.1 & 0.3 \\
\hline 2 & 0.75 & 3 & 0.0248 & 77.3 & 88.7 & 6 & 0.0480 & 16.3 & 20.6 & 5 & 0.0593 & 6.9 & 8.4 \\
\hline 2 & 0.90 & 3 & 0.0248 & 206.6 & 227.4 & 11 & 0.0459 & 105.4 & 128.2 & 12 & 0.0566 & 66.0 & 82.7 \\
\hline 3 & 0.50 & 1 & 0.0163 & 27.3 & 30.8 & 2 & 0.0463 & 1.8 & 1.6 & 2 & 0.0587 & 1.1 & 0.4 \\
\hline 3 & 0.75 & 1 & 0.0163 & 122.7 & 131.9 & 6 & 0.0422 & 20.7 & 26.1 & 5 & 0.0556 & 7.9 & 9.8 \\
\hline 3 & 0.90 & 1 & 0.0163 & 246.6 & 260.4 & 10 & 0.0404 & 118.0 & 141.9 & 12 & 0.0529 & 71.7 & 89.5 \\
\hline 4 & 0.50 & 1 & 0.0032 & 93.4 & 101.2 & 2 & 0.0400 & 2.3 & 2.3 & 2 & 0.0548 & 1.1 & 0.5 \\
\hline 4 & 0.75 & 1 & 0.0032 & 209.1 & 221.7 & 6 & 0.0360 & 27.0 & 33.8 & 5 & 0.0517 & 9.2 & 11.5 \\
\hline \multirow[t]{2}{*}{4} & 0.90 & 1 & 0.0032 & 300.5 & 315.9 & 9 & 0.0347 & 133.4 & 158.3 & 12 & 0.0491 & 78.3 & 97.3 \\
\hline & & & & & & \multicolumn{4}{|c|}{$\gamma_{0}=0.2$} & & & & \\
\hline 2 & 0.50 & 2 & 0.0528 & 10.9 & 13.2 & 2 & 0.1037 & 1.5 & 1.2 & 2 & 0.1240 & 1.1 & 0.3 \\
\hline 2 & 0.75 & 3 & 0.0492 & 78.8 & 90.4 & 6 & 0.0952 & 17.1 & 21.6 & 5 & 0.1177 & 7.2 & 8.9 \\
\hline 2 & 0.90 & 3 & 0.0492 & 208.5 & 229.4 & 11 & 0.0910 & 108.0 & 131.3 & 13 & 0.1119 & 68.4 & 85.9 \\
\hline 3 & 0.50 & 1 & 0.0323 & 27.9 & 31.5 & 2 & 0.0918 & 1.8 & 1.6 & 2 & 0.1164 & 1.1 & 0.4 \\
\hline 3 & 0.75 & 1 & 0.0323 & 124.5 & 133.8 & 6 & 0.0836 & 21.6 & 27.2 & 5 & 0.1103 & 8.3 & 10.4 \\
\hline 3 & 0.90 & 1 & 0.0323 & 248.2 & 262.1 & 10 & 0.0801 & 120.7 & 145.0 & 12 & 0.1050 & 74.2 & 92.4 \\
\hline 4 & 0.50 & 1 & 0.0064 & 94.7 & 102.6 & 2 & 0.0792 & 2.4 & 2.4 & 2 & 0.1086 & 1.2 & 0.5 \\
\hline 4 & 0.75 & 1 & 0.0064 & 210.7 & 223.4 & 6 & 0.0712 & 28.1 & 35.2 & 5 & 0.1025 & 9.7 & 12.2 \\
\hline \multirow[t]{2}{*}{4} & 0.90 & 1 & 0.0064 & 301.5 & 316.9 & 9 & 0.0686 & 136.1 & 161.5 & 12 & 0.0973 & 80.9 & 100.3 \\
\hline & & & & & & \multicolumn{4}{|c|}{$\gamma_{0}=0.3$} & & & & \\
\hline 2 & 0.50 & 2 & 0.0781 & 11.4 & 13.8 & 2 & 0.1536 & 1.6 & 1.3 & 2 & 0.1838 & 1.1 & 0.4 \\
\hline 2 & 0.75 & 3 & 0.0729 & 81.4 & 93.2 & 6 & 0.1409 & 18.3 & 23.2 & 5 & 0.1744 & 7.9 & 9.8 \\
\hline 2 & 0.90 & 3 & 0.0729 & 211.5 & 232.6 & 11 & 0.1346 & 112.2 & 136.0 & 13 & 0.1656 & 72.1 & 90.3 \\
\hline 3 & 0.50 & 1 & 0.0478 & 29.1 & 32.7 & 2 & 0.1358 & 1.9 & 1.8 & 2 & 0.1724 & 1.1 & 0.4 \\
\hline 3 & 0.75 & 1 & 0.0478 & 127.5 & 136.9 & 6 & 0.1235 & 23.1 & 29.1 & 5 & 0.1632 & 9.1 & 11.3 \\
\hline 3 & 0.90 & 1 & 0.0478 & 250.8 & 264.7 & 10 & 0.1184 & 125.0 & 149.9 & 13 & 0.1546 & 78.1 & 97.5 \\
\hline 4 & 0.50 & 1 & 0.0095 & 96.7 & 104.7 & 2 & 0.1170 & 2.5 & 2.6 & 2 & 0.1607 & 1.2 & 0.6 \\
\hline 4 & 0.75 & 1 & 0.0095 & 213.4 & 226.1 & 6 & 0.1052 & 30.0 & 37.4 & 6 & 0.1499 & 10.6 & 13.3 \\
\hline \multirow[t]{2}{*}{4} & 0.90 & 1 & 0.0095 & 303.1 & 318.6 & 9 & 0.1013 & 140.5 & 166.4 & 13 & 0.1431 & 85.0 & 105.6 \\
\hline & & & & & & \multicolumn{4}{|c|}{$\gamma_{0}=0.4$} & & & & \\
\hline 2 & 0.50 & 2 & 0.1024 & 12.2 & 14.7 & 2 & 0.2013 & 1.7 & 1.4 & 2 & 0.2413 & 1.1 & 0.4 \\
\hline 2 & 0.75 & 3 & 0.0955 & 84.9 & 97.1 & 6 & 0.1844 & 20.1 & 25.3 & 5 & 0.2287 & 8.8 & 10.9 \\
\hline 2 & 0.90 & 3 & 0.0955 & 215.5 & 236.9 & 11 & 0.1761 & 117.6 & 142.2 & 13 & 0.2171 & 77.0 & 96.2 \\
\hline 3 & 0.50 & 1 & 0.0625 & 30.6 & 34.4 & 2 & 0.1777 & 2.1 & 2.0 & 2 & 0.2261 & 1.2 & 0.5 \\
\hline 3 & 0.75 & 1 & 0.0625 & 131.6 & 141.2 & 6 & 0.1614 & 25.2 & 31.6 & 6 & 0.2115 & 10.1 & 12.7 \\
\hline 3 & 0.90 & 1 & 0.0625 & 254.2 & 268.2 & 10 & 0.1547 & 130.6 & 156.2 & 13 & 0.2023 & 83.3 & 103.6 \\
\hline 4 & 0.50 & 1 & 0.0124 & 99.6 & 107.8 & 2 & 0.1528 & 2.7 & 2.9 & 2 & 0.2104 & 1.2 & 0.6 \\
\hline 4 & 0.75 & 1 & 0.0124 & 217.1 & 229.9 & 6 & 0.1373 & 32.5 & 40.5 & 6 & 0.1961 & 11.8 & 14.8 \\
\hline \multirow[t]{2}{*}{4} & 0.90 & 1 & 0.0124 & 305.3 & 320.9 & 9 & 0.1321 & 146.3 & 172.8 & 13 & 0.1871 & 90.4 & 111.9 \\
\hline & & & & & & \multicolumn{4}{|c|}{$\gamma_{0}=0.5$} & & & & \\
\hline 2 & 0.50 & 2 & 0.1253 & 13.2 & 15.8 & 2 & 0.2465 & 1.8 & 1.6 & 2 & 0.2961 & 1.2 & 0.5 \\
\hline 2 & 0.75 & 3 & 0.1168 & 89.3 & 101.8 & 6 & 0.2256 & 22.3 & 28.1 & 6 & 0.2775 & 9.9 & 12.5 \\
\hline 2 & 0.90 & 3 & 0.1168 & 220.2 & 241.9 & 12 & 0.2139 & 123.9 & 150.2 & 14 & 0.2647 & 82.9 & 103.6 \\
\hline 3 & 0.50 & 1 & 0.0763 & 32.6 & 36.6 & 2 & 0.2171 & 2.3 & 2.2 & 2 & 0.2770 & 1.2 & 0.6 \\
\hline 3 & 0.75 & 1 & 0.0763 & 136.6 & 146.5 & 6 & 0.1971 & 27.9 & 34.9 & 6 & 0.2587 & 11.4 & 14.4 \\
\hline 3 & 0.90 & 1 & 0.0763 & 258.3 & 272.5 & 11 & 0.1872 & 137.2 & 164.6 & 13 & 0.2473 & 89.4 & 110.8 \\
\hline 4 & 0.50 & 1 & 0.0152 & 103.3 & 111.6 & 3 & 0.1789 & 3.0 & 3.2 & 2 & 0.2573 & 1.3 & 0.8 \\
\hline 4 & 0.75 & 1 & 0.0152 & 221.6 & 234.6 & 6 & 0.1672 & 35.8 & 44.4 & 6 & 0.2395 & 13.3 & 16.8 \\
\hline 4 & 0.90 & 1 & 0.0152 & 308.0 & 323.7 & 9 & 0.1610 & 153.0 & 180.3 & 13 & 0.2284 & 96.8 & 119.4 \\
\hline
\end{tabular}

Table 2. Zero-state performance. Values of $H^{-*}, L C L^{*}, A R L_{\mathrm{ZS}}, S D R L_{\mathrm{ZS}}$ for the lower-sided control charts, for different values of $n=\{5,10,15\}, p=\{2,3,4\}, \gamma_{0}=\{0.1,0.2,0.3,0.4,0.5\}, \tau=\{0.5,0.75,0.9\}$ and $A R L_{0}=370.4$ 


\begin{tabular}{|c|c|c|c|c|c|c|c|c|c|c|c|c|c|}
\hline \multirow{3}{*}{$p$} & \multirow{3}{*}{$\tau$} & \multicolumn{4}{|c|}{$n=5$} & \multicolumn{4}{|c|}{$n=10$} & \multicolumn{4}{|c|}{$n=15$} \\
\hline & & $H^{+*}$ & $U C L^{*}$ & $A R L_{\mathrm{ZS}}$ & $S D R L_{\mathrm{ZS}}$ & $H^{+*}$ & $U C L^{*}$ & $A R L_{\mathrm{ZS}}$ & $S D R L_{\mathrm{ZS}}$ & $H^{+*}$ & $U C L^{*}$ & $A R L_{\mathrm{ZS}}$ & $S D R L_{\mathrm{ZS}}$ \\
\hline & & & & & & \multicolumn{4}{|c|}{$\gamma_{0}=0.1$} & & & & \\
\hline 2 & 1.10 & 47 & 0.1729 & 74.7 & 97.8 & 31 & 0.1503 & 44.1 & 57.4 & 24 & 0.1403 & 31.4 & 40.7 \\
\hline 2 & 1.25 & 22 & 0.1671 & 17.9 & 22.4 & 12 & 0.1455 & 8.1 & 9.6 & 8 & 0.1359 & 5.1 & 5.8 \\
\hline 2 & 1.50 & 10 & 0.1607 & 5.4 & 5.9 & 5 & 0.1408 & 2.4 & 2.2 & 4 & 0.1329 & 1.7 & 1.2 \\
\hline 3 & 1.10 & 55 & 0.1569 & 88.5 & 116.0 & 33 & 0.1443 & 47.9 & 62.5 & 25 & 0.1366 & 33.3 & 43.3 \\
\hline 3 & 1.25 & 27 & 0.1515 & 23.7 & 30.0 & 13 & 0.1396 & 9.1 & 10.9 & 9 & 0.1325 & 5.6 & 6.3 \\
\hline 3 & 1.50 & 13 & 0.1456 & 7.4 & 8.5 & 6 & 0.1355 & 2.7 & 2.5 & 4 & 0.1290 & 1.8 & 1.4 \\
\hline 4 & 1.10 & 71 & 0.1356 & 112.5 & 147.6 & 35 & 0.1378 & 52.6 & 68.6 & 26 & 0.1327 & 35.5 & 46.2 \\
\hline 4 & 1.25 & 39 & 0.1310 & 35.8 & 45.9 & 14 & 0.1333 & 10.4 & 12.6 & 9 & 0.1285 & 6.0 & 7.0 \\
\hline \multirow[t]{2}{*}{4} & 1.50 & 21 & 0.1260 & 12.4 & 14.7 & 6 & 0.1287 & 3.0 & 3.0 & 4 & 0.1250 & 1.9 & 1.5 \\
\hline & & & & & & \multicolumn{4}{|c|}{$\gamma_{0}=0.2$} & & & & \\
\hline 2 & 1.10 & 47 & 0.3551 & 76.8 & 100.5 & 31 & 0.3061 & 46.1 & 60.1 & 25 & 0.2850 & 33.1 & 42.9 \\
\hline 2 & 1.25 & 22 & 0.3425 & 18.7 & 23.6 & 12 & 0.2958 & 8.6 & 10.3 & 9 & 0.2763 & 5.5 & 6.3 \\
\hline 2 & 1.50 & 10 & 0.3285 & 5.7 & 6.4 & 5 & 0.2857 & 2.6 & 2.4 & 4 & 0.2689 & 1.8 & 1.4 \\
\hline 3 & 1.10 & 55 & 0.3203 & 90.7 & 118.9 & 33 & 0.2932 & 50.0 & 65.3 & 26 & 0.2770 & 35.1 & 45.6 \\
\hline 3 & 1.25 & 28 & 0.3093 & 24.7 & 31.4 & 13 & 0.2832 & 9.7 & 11.8 & 9 & 0.2680 & 6.0 & 6.9 \\
\hline 3 & 1.50 & 14 & 0.2973 & 7.8 & 9.0 & 6 & 0.2744 & 2.9 & 2.7 & 4 & 0.2606 & 1.9 & 1.5 \\
\hline 4 & 1.10 & 71 & 0.2747 & 114.8 & 150.7 & 36 & 0.2796 & 54.8 & 71.6 & 27 & 0.2687 & 37.4 & 48.6 \\
\hline 4 & 1.25 & 40 & 0.2654 & 37.2 & 47.8 & 15 & 0.2704 & 11.1 & 13.5 & 10 & 0.2604 & 6.5 & 7.5 \\
\hline \multirow[t]{2}{*}{4} & 1.50 & 22 & 0.2552 & 13.0 & 15.6 & 6 & 0.2600 & 3.3 & 3.3 & 4 & 0.2521 & 2.0 & 1.7 \\
\hline & & & & & & \multicolumn{4}{|c|}{$\gamma_{0}=0.3$} & & & & \\
\hline 2 & 1.10 & 48 & 0.5587 & 80.4 & 105.3 & 32 & 0.4740 & 49.5 & 64.6 & 26 & 0.4384 & 36.0 & 46.7 \\
\hline 2 & 1.25 & 23 & 0.5372 & 20.3 & 25.6 & 13 & 0.4576 & 9.6 & 11.6 & 9 & 0.4233 & 6.2 & 7.2 \\
\hline 2 & 1.50 & 11 & 0.5146 & 6.2 & 7.1 & 6 & 0.4427 & 2.8 & 2.7 & 4 & 0.4110 & 1.9 & 1.6 \\
\hline 3 & 1.10 & 56 & 0.4978 & 94.6 & 124.0 & 34 & 0.4520 & 53.6 & 70.0 & 27 & 0.4250 & 38.1 & 49.6 \\
\hline 3 & 1.25 & 29 & 0.4796 & 26.6 & 33.9 & 14 & 0.4362 & 10.8 & 13.2 & 10 & 0.4111 & 6.7 & 7.8 \\
\hline 3 & 1.50 & 15 & 0.4604 & 8.6 & 10.0 & 6 & 0.4202 & 3.2 & 3.2 & 4 & 0.3974 & 2.1 & 1.8 \\
\hline 4 & 1.10 & 72 & 0.4210 & 119.0 & 156.2 & 37 & 0.4292 & 58.6 & 76.6 & 27 & 0.4107 & 40.5 & 52.8 \\
\hline 4 & 1.25 & 41 & 0.4062 & 39.8 & 51.2 & 16 & 0.4146 & 12.3 & 15.1 & 10 & 0.3970 & 7.2 & 8.6 \\
\hline \multirow[t]{2}{*}{4} & 1.50 & 23 & 0.3902 & 14.3 & 17.3 & 7 & 0.3994 & 3.6 & 3.8 & 5 & 0.3868 & 2.2 & 1.9 \\
\hline & & & & & & \multicolumn{4}{|c|}{$\gamma_{0}=0.4$} & & & & \\
\hline 2 & 1.10 & 49 & 0.7993 & 86.0 & 112.7 & 34 & 0.6618 & 54.4 & 71.0 & 27 & 0.6052 & 40.1 & 52.2 \\
\hline 2 & 1.25 & 24 & 0.7643 & 22.7 & 28.9 & 14 & 0.6365 & 11.0 & 13.5 & 10 & 0.5836 & 7.1 & 8.4 \\
\hline 2 & 1.50 & 12 & 0.7294 & 7.1 & 8.2 & 6 & 0.6111 & 3.3 & 3.3 & 4 & 0.5625 & 2.2 & 2.0 \\
\hline 3 & 1.10 & 57 & 0.6980 & 100.6 & 131.9 & 36 & 0.6268 & 58.8 & 76.9 & 28 & 0.5844 & 42.4 & 55.2 \\
\hline 3 & 1.25 & 30 & 0.6697 & 29.6 & 38.0 & 15 & 0.6027 & 12.4 & 15.3 & 11 & 0.5646 & 7.7 & 9.2 \\
\hline 3 & 1.50 & 16 & 0.6411 & 9.9 & 11.7 & 7 & 0.5808 & 3.7 & 3.8 & 5 & 0.5470 & 2.4 & 2.1 \\
\hline 4 & 1.10 & 73 & 0.5779 & 125.4 & 164.5 & 38 & 0.5904 & 64.0 & 83.8 & 29 & 0.5632 & 45.0 & 58.7 \\
\hline 4 & 1.25 & 43 & 0.5571 & 43.8 & 56.6 & 17 & 0.5692 & 14.1 & 17.6 & 11 & 0.5431 & 8.4 & 10.1 \\
\hline \multirow[t]{2}{*}{4} & 1.50 & 24 & 0.5332 & 16.3 & 20.0 & 8 & 0.5484 & 4.2 & 4.5 & 5 & 0.5259 & 2.5 & 2.4 \\
\hline & & & & & & \multicolumn{4}{|c|}{$\gamma_{0}=0.5$} & & & & \\
\hline 2 & 1.10 & 49 & 1.0991 & 94.1 & 123.2 & 35 & 0.8774 & 60.8 & 79.5 & 28 & 0.7908 & 45.4 & 59.2 \\
\hline 2 & 1.25 & 25 & 1.0432 & 26.3 & 33.8 & 15 & 0.8401 & 13.0 & 16.2 & 11 & 0.7607 & 8.5 & 10.2 \\
\hline 2 & 1.50 & 13 & 0.9890 & 8.5 & 10.1 & 7 & 0.8057 & 3.9 & 4.1 & 5 & 0.7343 & 2.6 & 2.4 \\
\hline 3 & 1.10 & 58 & 0.9307 & 109.3 & 143.2 & 37 & 0.8224 & 65.7 & 85.8 & 29 & 0.7592 & 48.0 & 62.6 \\
\hline 3 & 1.25 & 32 & 0.8900 & 34.3 & 44.2 & 16 & 0.7879 & 14.7 & 18.4 & 12 & 0.7319 & 9.2 & 11.1 \\
\hline 3 & 1.50 & 17 & 0.8462 & 11.9 & 14.5 & 8 & 0.7585 & 4.4 & 4.8 & 5 & 0.7037 & 2.8 & 2.7 \\
\hline 4 & 1.10 & 73 & 0.7468 & 134.4 & 176.3 & 39 & 0.7671 & 71.3 & 93.3 & 31 & 0.7286 & 50.9 & 66.4 \\
\hline 4 & 1.25 & 45 & 0.7195 & 49.9 & 64.7 & 18 & 0.7373 & 16.7 & 21.1 & 12 & 0.7003 & 10.0 & 12.3 \\
\hline 4 & 1.50 & 26 & 0.6877 & 19.5 & 24.4 & 9 & 0.7098 & 5.1 & 5.7 & 6 & 0.6788 & 3.0 & 3.0 \\
\hline
\end{tabular}

Table 3. Zero-state performance. Values of $H^{+*}, U C L^{*}, A R L_{\mathrm{ZS}}, S D R L_{\mathrm{ZS}}$ for the upper-sided control charts, for different values of $n=\{5,10,15\}, p=\{2,3,4\}, \gamma_{0}=\{0.1,0.2,0.3,0.4,0.5\}, \tau=\{1.1,1.25,1.5\}$ and $A R L_{0}=370.4$ 


\begin{tabular}{|c|c|c|c|c|c|c|c|c|c|c|c|c|c|}
\hline \multirow{3}{*}{$p$} & \multirow{3}{*}{$\tau$} & \multicolumn{4}{|c|}{$n=5$} & \multicolumn{4}{|c|}{$n=10$} & \multicolumn{4}{|c|}{$n=15$} \\
\hline & & $H^{-*}$ & $L C L^{*}$ & $A R L_{\mathrm{SS}}$ & $S D R L_{\mathrm{SS}}$ & $H^{-*}$ & $L C L^{*}$ & $A R L_{\mathrm{SS}}$ & $S D R L_{\mathrm{SS}}$ & $H^{-*}$ & $L C L^{*}$ & $A R L_{\mathrm{SS}}$ & $S D R L_{\mathrm{SS}}$ \\
\hline & & & & & & \multicolumn{4}{|c|}{$\gamma_{0}=0.1$} & & & & \\
\hline 2 & 0.50 & 1 & 0.0303 & 13.5 & 12.3 & 1 & 0.0555 & 2.7 & 1.4 & 2 & 0.0627 & 2.0 & 0.5 \\
\hline 2 & 0.75 & 1 & 0.0303 & 82.8 & 81.9 & 2 & 0.0526 & 21.4 & 20.1 & 2 & 0.0627 & 10.2 & 8.9 \\
\hline 2 & 0.90 & 1 & 0.0303 & 211.0 & 210.4 & 3 & 0.0510 & 115.5 & 114.3 & 4 & 0.0604 & 76.5 & 75.1 \\
\hline 3 & 0.50 & 1 & 0.0165 & 30.9 & 29.8 & 1 & 0.0495 & 3.1 & 1.9 & 2 & 0.0589 & 2.1 & 0.5 \\
\hline 3 & 0.75 & 1 & 0.0165 & 127.2 & 126.3 & 2 & 0.0466 & 26.2 & 24.9 & 2 & 0.0589 & 11.5 & 10.2 \\
\hline 3 & 0.90 & 1 & 0.0165 & 249.3 & 248.7 & 3 & 0.0451 & 127.6 & 126.6 & 4 & 0.0566 & 82.3 & 80.9 \\
\hline 4 & 0.50 & 1 & 0.0033 & 97.9 & 97.0 & 1 & 0.0431 & 3.8 & 2.6 & 1 & 0.0575 & 2.2 & 0.7 \\
\hline 4 & 0.75 & 1 & 0.0033 & 212.5 & 211.8 & 2 & 0.0403 & 32.9 & 31.7 & 2 & 0.0550 & 13.2 & 11.8 \\
\hline \multirow[t]{2}{*}{4} & 0.90 & 1 & 0.0033 & 302.1 & 301.5 & 3 & 0.0387 & 142.3 & 141.3 & 4 & 0.0528 & 88.9 & 87.6 \\
\hline & & & & & & \multicolumn{4}{|c|}{$\gamma_{0}=0.2$} & & & & \\
\hline 2 & 0.50 & 1 & 0.0600 & 13.8 & 12.7 & 1 & 0.1103 & 2.8 & 1.5 & 2 & 0.1245 & 2.1 & 0.5 \\
\hline 2 & 0.75 & 1 & 0.0600 & 84.4 & 83.5 & 2 & 0.1043 & 22.3 & 20.9 & 2 & 0.1245 & 10.7 & 9.4 \\
\hline 2 & 0.90 & 1 & 0.0600 & 212.9 & 212.2 & 3 & 0.1011 & 118.1 & 117.0 & 4 & 0.1198 & 79.0 & 77.6 \\
\hline 3 & 0.50 & 1 & 0.0327 & 31.6 & 30.5 & 1 & 0.0982 & 3.2 & 2.0 & 2 & 0.1169 & 2.1 & 0.6 \\
\hline 3 & 0.75 & 1 & 0.0327 & 129.0 & 128.1 & 2 & 0.0924 & 27.2 & 25.9 & 2 & 0.1169 & 12.1 & 10.7 \\
\hline 3 & 0.90 & 1 & 0.0327 & 250.9 & 250.3 & 3 & 0.0893 & 130.4 & 129.3 & 4 & 0.1124 & 84.9 & 83.5 \\
\hline 4 & 0.50 & 1 & 0.0066 & 99.1 & 98.2 & 1 & 0.0853 & 4.0 & 2.7 & 1 & 0.1141 & 2.2 & 0.7 \\
\hline 4 & 0.75 & 1 & 0.0066 & 214.1 & 213.4 & 2 & 0.0797 & 34.2 & 32.9 & 2 & 0.1091 & 13.8 & 12.4 \\
\hline \multirow[t]{2}{*}{4} & 0.90 & 1 & 0.0066 & 303.0 & 302.5 & 3 & 0.0767 & 145.1 & 144.0 & 4 & 0.1046 & 91.6 & 90.2 \\
\hline & & & & & & \multicolumn{4}{|c|}{$\gamma_{0}=0.3$} & & & & \\
\hline 2 & 0.50 & 1 & 0.0889 & 14.4 & 13.3 & 1 & 0.1634 & 2.9 & 1.6 & 2 & 0.1846 & 2.1 & 0.5 \\
\hline 2 & 0.75 & 1 & 0.0889 & 87.0 & 86.1 & 2 & 0.1545 & 23.7 & 22.4 & 2 & 0.1846 & 11.5 & 10.2 \\
\hline 2 & 0.90 & 1 & 0.0889 & 215.9 & 215.2 & 3 & 0.1497 & 122.3 & 121.2 & 4 & 0.1776 & 82.9 & 81.5 \\
\hline 3 & 0.50 & 1 & 0.0484 & 32.7 & 31.7 & 1 & 0.1453 & 3.4 & 2.1 & 2 & 0.1732 & 2.2 & 0.6 \\
\hline 3 & 0.75 & 1 & 0.0484 & 131.9 & 131.1 & 2 & 0.1367 & 28.9 & 27.6 & 2 & 0.1732 & 13.0 & 11.7 \\
\hline 3 & 0.90 & 1 & 0.0484 & 253.4 & 252.8 & 3 & 0.1320 & 134.6 & 133.6 & 4 & 0.1663 & 89.0 & 87.6 \\
\hline 4 & 0.50 & 1 & 0.0098 & 101.2 & 100.3 & 1 & 0.1261 & 4.1 & 2.9 & 1 & 0.1689 & 2.3 & 0.8 \\
\hline 4 & 0.75 & 1 & 0.0098 & 216.7 & 216.0 & 2 & 0.1178 & 36.2 & 34.9 & 2 & 0.1614 & 14.8 & 13.5 \\
\hline \multirow[t]{2}{*}{4} & 0.90 & 1 & 0.0098 & 304.7 & 304.1 & 3 & 0.1133 & 149.4 & 148.4 & 4 & 0.1547 & 95.8 & 94.5 \\
\hline & & & & & & \multicolumn{4}{|c|}{$\gamma_{0}=0.4$} & & & & \\
\hline 2 & 0.50 & 1 & 0.1166 & 15.2 & 14.1 & 1 & 0.2143 & 3.0 & 1.7 & 2 & 0.2423 & 2.1 & 0.6 \\
\hline 2 & 0.75 & 1 & 0.1166 & 90.6 & 89.7 & 2 & 0.2025 & 25.7 & 24.4 & 2 & 0.2423 & 12.7 & 11.3 \\
\hline 2 & 0.90 & 1 & 0.1166 & 219.8 & 219.1 & 3 & 0.1962 & 127.8 & 126.7 & 4 & 0.2330 & 88.1 & 86.7 \\
\hline 3 & 0.50 & 1 & 0.0633 & 34.4 & 33.3 & 1 & 0.1902 & 3.6 & 2.3 & 1 & 0.2372 & 2.2 & 0.8 \\
\hline 3 & 0.75 & 1 & 0.0633 & 136.0 & 135.1 & 2 & 0.1788 & 31.3 & 30.0 & 2 & 0.2271 & 14.3 & 13.0 \\
\hline 3 & 0.90 & 1 & 0.0633 & 256.8 & 256.2 & 3 & 0.1727 & 140.2 & 139.2 & 4 & 0.2179 & 94.3 & 93.0 \\
\hline 4 & 0.50 & 1 & 0.0128 & 104.1 & 103.2 & 1 & 0.1648 & 4.4 & 3.2 & 1 & 0.2213 & 2.3 & 0.9 \\
\hline 4 & 0.75 & 1 & 0.0128 & 220.3 & 219.6 & 2 & 0.1539 & 39.0 & 37.8 & 2 & 0.2114 & 16.3 & 15.0 \\
\hline \multirow[t]{2}{*}{4} & 0.90 & 1 & 0.0128 & 306.8 & 306.3 & 3 & 0.1480 & 155.1 & 154.1 & 4 & 0.2024 & 101.3 & 100.0 \\
\hline & & & & & & \multicolumn{4}{|c|}{$\gamma_{0}=0.5$} & & & & \\
\hline 2 & 0.50 & 1 & 0.1427 & 16.3 & 15.2 & 1 & 0.2627 & 3.2 & 2.0 & 2 & 0.2974 & 2.2 & 0.7 \\
\hline 2 & 0.75 & 1 & 0.1427 & 95.0 & 94.1 & 2 & 0.2480 & 28.3 & 27.0 & 2 & 0.2974 & 14.2 & 12.8 \\
\hline 2 & 0.90 & 1 & 0.1427 & 224.4 & 223.8 & 3 & 0.2401 & 134.1 & 133.1 & 4 & 0.2857 & 94.2 & 92.8 \\
\hline 3 & 0.50 & 1 & 0.0773 & 36.4 & 35.4 & 1 & 0.2327 & 3.8 & 2.6 & 1 & 0.2909 & 2.3 & 0.9 \\
\hline 3 & 0.75 & 1 & 0.0773 & 141.0 & 140.2 & 2 & 0.2185 & 34.3 & 33.0 & 2 & 0.2782 & 16.0 & 14.6 \\
\hline 3 & 0.90 & 1 & 0.0773 & 260.8 & 260.2 & 3 & 0.2110 & 146.8 & 145.8 & 4 & 0.2668 & 100.6 & 99.3 \\
\hline 4 & 0.50 & 1 & 0.0155 & 107.8 & 106.9 & 1 & 0.2011 & 4.8 & 3.6 & 1 & 0.2709 & 2.4 & 1.0 \\
\hline 4 & 0.75 & 1 & 0.0155 & 224.7 & 224.1 & 2 & 0.1876 & 42.6 & 41.3 & 2 & 0.2585 & 18.2 & 16.9 \\
\hline 4 & 0.90 & 1 & 0.0155 & 309.5 & 308.9 & 3 & 0.1805 & 161.7 & 160.8 & 4 & 0.2474 & 107.9 & 106.6 \\
\hline
\end{tabular}

Table 4. Steady-state performance. Values of $H^{-*}, L C L^{*}, A R L_{\mathrm{SS}}, S D R L_{\mathrm{SS}}$ for the lower-sided control charts, for different values of $n=\{5,10,15\}, p=\{2,3,4\}, \gamma_{0}=\{0.1,0.2,0.3,0.4,0.5\}, \tau=\{0.5,0.75,0.9\}$ and $A R L_{0}=370.4$ 


\begin{tabular}{|c|c|c|c|c|c|c|c|c|c|c|c|c|c|}
\hline \multirow{3}{*}{$p$} & \multirow{3}{*}{$\tau$} & \multicolumn{4}{|c|}{$n=5$} & \multicolumn{4}{|c|}{$n=10$} & \multicolumn{4}{|c|}{$n=15$} \\
\hline & & $H^{+*}$ & $U C L^{*}$ & $A R L_{\mathrm{SS}}$ & $S D R L_{\mathrm{SS}}$ & $H^{+*}$ & $U C L^{*}$ & $A R L_{\mathrm{SS}}$ & $S D R L_{\mathrm{SS}}$ & $H^{+*}$ & $U C L^{*}$ & $A R L_{\mathrm{SS}}$ & $S D R L_{\mathrm{SS}}$ \\
\hline & & & & & & \multicolumn{4}{|c|}{$\gamma_{0}=0.1$} & & & & \\
\hline 2 & 1.10 & 24 & 0.1660 & 94.0 & 91.3 & 14 & 0.1454 & 58.6 & 56.0 & 11 & 0.1365 & 43.3 & 40.8 \\
\hline 2 & 1.25 & 13 & 0.1614 & 27.1 & 24.4 & 7 & 0.1419 & 13.1 & 11.1 & 5 & 0.1334 & 8.7 & 6.9 \\
\hline 2 & 1.50 & 7 & 0.1565 & 9.2 & 7.2 & 4 & 0.1390 & 4.4 & 2.8 & 3 & 0.1312 & 3.1 & 1.6 \\
\hline 3 & 1.10 & 29 & 0.1501 & 109.4 & 106.8 & 15 & 0.1394 & 63.2 & 60.6 & 12 & 0.1330 & 45.7 & 43.1 \\
\hline 3 & 1.25 & 16 & 0.1457 & 34.9 & 32.0 & 7 & 0.1356 & 14.6 & 12.6 & 5 & 0.1295 & 9.3 & 7.6 \\
\hline 3 & 1.50 & 9 & 0.1412 & 12.4 & 10.2 & 4 & 0.1327 & 4.8 & 3.3 & 3 & 0.1273 & 3.3 & 1.8 \\
\hline 4 & 1.10 & 45 & 0.1297 & 135.4 & 133.4 & 17 & 0.1332 & 68.7 & 65.9 & 12 & 0.1290 & 48.4 & 45.8 \\
\hline 4 & 1.25 & 26 & 0.1258 & 50.9 & 47.4 & 8 & 0.1295 & 16.5 & 14.3 & 6 & 0.1262 & 10.0 & 8.2 \\
\hline \multirow[t]{2}{*}{4} & 1.50 & 15 & 0.1215 & 19.9 & 17.2 & 5 & 0.1271 & 5.4 & 3.8 & 3 & 0.1233 & 3.5 & 2.0 \\
\hline & & & & & & \multicolumn{4}{|c|}{$\gamma_{0}=0.2$} & & & & \\
\hline 2 & 1.10 & 23 & 0.3393 & 96.2 & 93.6 & 14 & 0.2955 & 60.9 & 58.3 & 11 & 0.2766 & 45.3 & 42.9 \\
\hline 2 & 1.25 & 13 & 0.3301 & 28.2 & 25.5 & 7 & 0.2882 & 13.9 & 11.9 & 5 & 0.2699 & 9.3 & 7.5 \\
\hline 2 & 1.50 & 7 & 0.3195 & 9.7 & 7.7 & 4 & 0.2819 & 4.6 & 3.1 & 3 & 0.2653 & 3.3 & 1.8 \\
\hline 3 & 1.10 & 30 & 0.3061 & 111.7 & 109.1 & 16 & 0.2834 & 65.6 & 62.9 & 12 & 0.2691 & 47.8 & 45.3 \\
\hline 3 & 1.25 & 17 & 0.2972 & 36.3 & 33.3 & 8 & 0.2761 & 15.5 & 13.3 & 6 & 0.2633 & 9.9 & 8.0 \\
\hline 3 & 1.50 & 10 & 0.2884 & 13.0 & 10.7 & 4 & 0.2684 & 5.1 & 3.5 & 3 & 0.2571 & 3.5 & 2.0 \\
\hline 4 & 1.10 & 45 & 0.2623 & 137.8 & 135.9 & 17 & 0.2696 & 71.1 & 68.4 & 12 & 0.2605 & 50.5 & 48.0 \\
\hline 4 & 1.25 & 26 & 0.2540 & 52.6 & 49.2 & 8 & 0.2617 & 17.5 & 15.3 & 6 & 0.2547 & 10.7 & 8.8 \\
\hline \multirow[t]{2}{*}{4} & 1.50 & 16 & 0.2461 & 20.9 & 18.0 & 5 & 0.2565 & 5.8 & 4.1 & 3 & 0.2485 & 3.7 & 2.2 \\
\hline & & & & & & \multicolumn{4}{|c|}{$\gamma_{0}=0.3$} & & & & \\
\hline 2 & 1.10 & 24 & 0.5315 & 100.1 & 97.5 & 15 & 0.4568 & 64.8 & 62.2 & 12 & 0.4251 & 48.7 & 46.2 \\
\hline 2 & 1.25 & 13 & 0.5143 & 30.2 & 27.5 & 7 & 0.4432 & 15.2 & 13.2 & 6 & 0.4154 & 10.2 & 8.3 \\
\hline 2 & 1.50 & 8 & 0.5001 & 10.5 & 8.5 & 4 & 0.4326 & 5.1 & 3.5 & 3 & 0.4051 & 3.6 & 2.1 \\
\hline 3 & 1.10 & 30 & 0.4732 & 115.8 & 113.3 & 16 & 0.4351 & 69.6 & 67.0 & 12 & 0.4113 & 51.3 & 48.8 \\
\hline 3 & 1.25 & 17 & 0.4582 & 38.7 & 35.7 & 8 & 0.4231 & 17.0 & 14.8 & 6 & 0.4018 & 11.0 & 9.1 \\
\hline 3 & 1.50 & 10 & 0.4435 & 14.2 & 11.9 & 5 & 0.4144 & 5.7 & 4.0 & 3 & 0.3916 & 3.8 & 2.3 \\
\hline 4 & 1.10 & 45 & 0.4005 & 142.0 & 140.2 & 17 & 0.4121 & 75.4 & 72.7 & 13 & 0.3982 & 54.2 & 51.6 \\
\hline 4 & 1.25 & 27 & 0.3879 & 55.7 & 52.2 & 9 & 0.4013 & 19.1 & 16.9 & 6 & 0.3877 & 11.8 & 9.9 \\
\hline \multirow[t]{2}{*}{4} & 1.50 & 16 & 0.3743 & 22.7 & 19.8 & 5 & 0.3908 & 6.4 & 4.7 & 4 & 0.3819 & 4.0 & 2.5 \\
\hline & & & & & & \multicolumn{4}{|c|}{$\gamma_{0}=0.4$} & & & & \\
\hline 2 & 1.10 & 24 & 0.7527 & 106.0 & 103.4 & 15 & 0.6330 & 70.3 & 67.7 & 12 & 0.5839 & 53.5 & 51.0 \\
\hline 2 & 1.25 & 13 & 0.7245 & 33.3 & 30.6 & 8 & 0.6156 & 17.2 & 15.1 & 6 & 0.5692 & 11.6 & 9.7 \\
\hline 2 & 1.50 & 8 & 0.7015 & 11.9 & 9.8 & 4 & 0.5955 & 5.8 & 4.2 & 3 & 0.5536 & 4.0 & 2.5 \\
\hline 3 & 1.10 & 30 & 0.6582 & 121.9 & 119.5 & 16 & 0.5991 & 75.4 & 72.8 & 13 & 0.5644 & 56.3 & 53.7 \\
\hline 3 & 1.25 & 17 & 0.6348 & 42.4 & 39.5 & 8 & 0.5807 & 19.2 & 17.0 & 6 & 0.5484 & 12.5 & 10.6 \\
\hline 3 & 1.50 & 10 & 0.6121 & 16.1 & 13.7 & 5 & 0.5676 & 6.4 & 4.7 & 4 & 0.5396 & 4.3 & 2.7 \\
\hline 4 & 1.10 & 45 & 0.5467 & 148.3 & 146.7 & 17 & 0.5638 & 81.4 & 78.8 & 13 & 0.5429 & 59.4 & 56.8 \\
\hline 4 & 1.25 & 27 & 0.5280 & 60.4 & 57.0 & 9 & 0.5476 & 21.6 & 19.3 & 6 & 0.5272 & 13.5 & 11.6 \\
\hline \multirow[t]{2}{*}{4} & 1.50 & 17 & 0.5103 & 25.5 & 22.5 & 5 & 0.5319 & 7.3 & 5.6 & 4 & 0.5186 & 4.6 & 3.0 \\
\hline & & & & & & \multicolumn{4}{|c|}{$\gamma_{0}=0.5$} & & & & \\
\hline 2 & 1.10 & 23 & 1.0174 & 114.1 & 111.7 & 15 & 0.8317 & 77.3 & 74.8 & 12 & 0.7580 & 59.6 & 57.1 \\
\hline 2 & 1.25 & 14 & 0.9802 & 37.8 & 35.0 & 8 & 0.8054 & 20.0 & 17.8 & 6 & 0.7363 & 13.6 & 11.6 \\
\hline 2 & 1.50 & 8 & 0.9378 & 13.9 & 11.8 & 5 & 0.7851 & 6.7 & 5.0 & 4 & 0.7232 & 4.6 & 3.0 \\
\hline 3 & 1.10 & 29 & 0.8658 & 130.5 & 128.3 & 16 & 0.7798 & 82.8 & 80.3 & 13 & 0.7289 & 62.6 & 60.1 \\
\hline 3 & 1.25 & 17 & 0.8324 & 48.0 & 45.0 & 9 & 0.7573 & 22.3 & 20.0 & 7 & 0.7104 & 14.6 & 12.5 \\
\hline 3 & 1.50 & 11 & 0.8047 & 18.9 & 16.5 & 5 & 0.7335 & 7.6 & 5.9 & 4 & 0.6930 & 4.9 & 3.4 \\
\hline 4 & 1.10 & 44 & 0.7009 & 157.0 & 155.6 & 18 & 0.7293 & 89.2 & 86.6 & 13 & 0.6974 & 66.0 & 63.5 \\
\hline 4 & 1.25 & 28 & 0.6776 & 67.3 & 64.0 & 10 & 0.7078 & 25.1 & 22.7 & 7 & 0.6795 & 15.8 & 13.7 \\
\hline 4 & 1.50 & 18 & 0.6541 & 29.8 & 26.7 & 6 & 0.6884 & 8.7 & 6.8 & 4 & 0.6627 & 5.3 & 3.7 \\
\hline
\end{tabular}

Table 5. Steady-state performance. Values of $H^{+*}, U C L^{*}, A R L_{\mathrm{SS}}, S D R L_{\mathrm{SS}}$ for the upper-sided control charts, for different values of $n=\{5,10,15\}, p=\{2,3,4\}, \gamma_{0}=\{0.1,0.2,0.3,0.4,0.5\}, \tau=\{1.1,1.25,1.5\}$ and $A R L_{0}=370.4$ 


\begin{tabular}{|c|c|c|c|c|c|c|c|c|c|}
\hline \multirow[b]{2}{*}{$\tau$} & \multicolumn{3}{|c|}{$p=2$} & \multicolumn{3}{|c|}{$p=3$} & \multicolumn{3}{|c|}{$p=4$} \\
\hline & $n=5$ & $n=10$ & $n=15$ & $n=5$ & $n=10$ & $n=15$ & $n=5$ & $n=10$ & $n=15$ \\
\hline & & & & & $\gamma_{0}=0$ & & & & \\
\hline 0.50 & 78 & 72 & 45 & 71 & 75 & 51 & 50 & 78 & 57 \\
\hline 0.75 & 51 & 69 & 72 & 41 & 67 & 72 & 25 & 65 & 72 \\
\hline 0.90 & 24 & 41 & 49 & 18 & 38 & 48 & 10 & 36 & 46 \\
\hline 1.10 & 37 & 46 & 50 & 34 & 44 & 49 & 29 & 43 & 48 \\
\hline 1.25 & 50 & 55 & 55 & 47 & 54 & 55 & 42 & 54 & 55 \\
\hline \multirow[t]{2}{*}{1.50} & 48 & 46 & 40 & 48 & 46 & 41 & 45 & 47 & 42 \\
\hline & & & & \multicolumn{3}{|c|}{$\gamma_{0}=0.2$} & & & \\
\hline 0.50 & 78 & 72 & 46 & 70 & 76 & 52 & 49 & 78 & 58 \\
\hline 0.75 & 51 & 69 & 72 & 41 & 67 & 72 & 25 & 65 & 71 \\
\hline 0.90 & 24 & 40 & 48 & 18 & 38 & 47 & 10 & 35 & 46 \\
\hline 1.10 & 37 & 45 & 50 & 33 & 44 & 49 & 28 & 43 & 48 \\
\hline 1.25 & 50 & 55 & 56 & 47 & 54 & 56 & 42 & 54 & 56 \\
\hline \multirow[t]{2}{*}{1.50} & 49 & 47 & 42 & 48 & 48 & 43 & 45 & 48 & 44 \\
\hline & & & & \multicolumn{3}{|c|}{$\gamma_{0}=0.3$} & & & \\
\hline 0.50 & 78 & 73 & 49 & 70 & 76 & 54 & 49 & 79 & 59 \\
\hline 0.75 & 50 & 68 & 72 & 40 & 66 & 71 & 24 & 64 & 71 \\
\hline 0.90 & 23 & 39 & 47 & 17 & 37 & 46 & 10 & 34 & 45 \\
\hline 1.10 & 36 & 44 & 49 & 33 & 43 & 48 & 28 & 42 & 47 \\
\hline 1.25 & 49 & 55 & 56 & 46 & 54 & 56 & 41 & 53 & 56 \\
\hline \multirow[t]{2}{*}{1.50} & 50 & 48 & 44 & 48 & 49 & 45 & 45 & 50 & 46 \\
\hline & & & & \multicolumn{3}{|c|}{$\gamma_{0}=0.4$} & & & \\
\hline 0.50 & 77 & 74 & 52 & 69 & 76 & 57 & 48 & 79 & 62 \\
\hline 0.75 & 49 & 67 & 71 & 39 & 65 & 71 & 23 & 63 & 70 \\
\hline 0.90 & 22 & 38 & 46 & 17 & 36 & 45 & 9 & 33 & 43 \\
\hline 1.10 & 35 & 43 & 48 & 32 & 42 & 47 & 27 & 41 & 46 \\
\hline 1.25 & 49 & 55 & 57 & 46 & 54 & 56 & 41 & 53 & 56 \\
\hline \multirow[t]{2}{*}{1.50} & 51 & 51 & 47 & 49 & 51 & 48 & 46 & 51 & 49 \\
\hline & & & & \multicolumn{3}{|c|}{$\gamma_{0}=0.5$} & & & \\
\hline 0.50 & 76 & 74 & 55 & 68 & 77 & 59 & 47 & 79 & 64 \\
\hline 0.75 & 48 & 66 & 70 & 38 & 64 & 70 & 23 & 62 & 69 \\
\hline 0.90 & 22 & 37 & 45 & 16 & 34 & 43 & 9 & 32 & 42 \\
\hline 1.10 & 34 & 42 & 47 & 31 & 41 & 46 & 26 & 39 & 45 \\
\hline 1.25 & 49 & 55 & 57 & 46 & 54 & 57 & 40 & 53 & 56 \\
\hline 1.50 & 53 & 53 & 51 & 51 & 53 & 51 & 46 & 53 & 52 \\
\hline
\end{tabular}

Table 6. Comparison vs. the Shewhart MCV control chart. $\Delta_{E}$ values of the Synthetic MCV control charts for different values of $p=\{2,3,4\}, n=\{5,10,15\}, \gamma_{0}=\{0.1,0.2,0.3,0.4,0.5\}, \tau=\{0.5,0.75,0.9,1.1,1.25,1.5\}$ and $A R L_{0}=370.4$. 


\begin{tabular}{|c|c|c|c|c|}
\hline$\Omega$ & $p$ & $n=5$ & $n=10$ & $n=15$ \\
\hline (D) & 2 & $(3,0.0248,96.5)$ & $\begin{array}{c}\gamma_{0}=0.1 \\
(7,0.0475,42.9)\end{array}$ & $(9,0.0575,28.3)$ \\
\hline (I) & 2 & $(27,0.1687,19.8)$ & $(21,0.1484,11.9)$ & $(19,0.1394,8.9)$ \\
\hline (D) & 3 & $(1,0.0163,131.3)$ & $(7,0.0416,48.0)$ & $(9,0.0538,30.3)$ \\
\hline (I) & 3 & $(30,0.1524,23.9)$ & $(22,0.1423,12.8)$ & $(20,0.1357,9.4)$ \\
\hline (D) & 4 & $(1,0.0032,203.0)$ & $(6,0.0360,54.7)$ & $(8,0.0503,32.7)$ \\
\hline (I) & 4 & $(37,0.1306,31.9)$ & $(23,0.1358,14.0)$ & $(20,0.1317,9.9)$ \\
\hline (D) & 2 & $(3,0.0492,97.7)$ & $\begin{array}{c}\gamma_{0}=0.2 \\
(7,0.0941,43.9)\end{array}$ & $(9,0.1141,29.1)$ \\
\hline (I) & 2 & $(27,0.3460,20.4)$ & $(21,0.3020,12.4)$ & $(19,0.2827,9.3)$ \\
\hline (D) & 3 & $(1,0.0323,132.7)$ & $(7,0.0825,49.1)$ & $(9,0.1066,31.2)$ \\
\hline (I) & 3 & $(30,0.3105,24.6)$ & $(22,0.2889,13.4)$ & $(20,0.2749,9.8)$ \\
\hline (D) & 4 & $(1,0.0064,204.4)$ & $(6,0.0712,55.9)$ & $(9,0.0990,33.6)$ \\
\hline (I) & 4 & $(37,0.2641,32.8)$ & $(23,0.2750,14.6)$ & $(20,0.2663,10.3)$ \\
\hline & & & $\gamma_{0}=0.3$ & \\
\hline (D) & 2 & $(3,0.0729,99.7)$ & $(7,0.1392,45.5)$ & $(9,0.1689,30.4)$ \\
\hline (I) & 2 & $(27,0.5420,21.5)$ & $(21,0.4664,13.2)$ & $(19,0.4340,10.0)$ \\
\hline (D) & 3 & $(1,0.0478,134.9)$ & $(7,0.1219,50.9)$ & $(9,0.1578,32.6)$ \\
\hline (I) & 3 & $(30,0.4805,25.9)$ & $(22,0.4444,14.3)$ & $(19,0.4202,10.5)$ \\
\hline (D) & 4 & $(1,0.0095,206.6)$ & $(6,0.1052,57.8)$ & $(9,0.1463,35.1)$ \\
\hline (I) & 4 & $(37,0.4034,34.5)$ & $(23,0.4210,15.5)$ & $(20,0.4067,11.1)$ \\
\hline (D) & 2 & $(3,0.0955,102.5)$ & $\begin{array}{c}\gamma_{0}=0.4 \\
(8,0.1804,47.7)\end{array}$ & $(9,0.2214,32.2)$ \\
\hline (I) & 2 & $(26,0.7682,23.2)$ & $(21,0.6482,14.5)$ & $(19,0.5977,11.0)$ \\
\hline (D) & 3 & $(1,0.0625,138.0)$ & $(7,0.1593,53.2)$ & $(9,0.2066,34.4)$ \\
\hline (I) & 3 & $(29,0.6682,27.9)$ & $(21,0.6121,15.6)$ & $(19,0.5763,11.5)$ \\
\hline (D) & 4 & $(1,0.0124,209.6)$ & $(6,0.1373,60.4)$ & $(9,0.1913,37.0)$ \\
\hline (I) & 4 & $(37,0.5510,37.2)$ & $(22,0.5761,17.0)$ & $(20,0.5556,12.2)$ \\
\hline & & & $\gamma_{0}=0.5$ & \\
\hline (D) & 2 & $(3,0.1168,105.8)$ & $(8,0.2206,50.3)$ & $(9,0.2712,34.3)$ \\
\hline (I) & 2 & $(25,1.0432,25.7)$ & $(20,0.8529,16.1)$ & $(19,0.7785,12.3)$ \\
\hline (D) & 3 & $(1,0.0763,141.9)$ & $(7,0.1945,56.1)$ & $(9,0.2526,36.7)$ \\
\hline (I) & 3 & $(29,0.8832,31.0)$ & $(21,0.7992,17.4)$ & $(19,0.7463,12.9)$ \\
\hline (D) & 4 & $(1,0.0152,213.5)$ & $(6,0.1672,63.5)$ & $(9,0.2336,39.4)$ \\
\hline (I) & 4 & $(37,0.7082,41.2)$ & $(22,0.7452,19.0)$ & $(19,0.7142,13.6)$ \\
\hline
\end{tabular}

Table 7. Zero-state performance. Optimal couples $\left(H^{-*}, L C L^{*}\right)$ for $\Omega=[0.5,1)$ and $\left(H^{+*}, U C L^{*}\right)$ for $\Omega=$ $(1,2]$ (two first values of each column) and out-of-control $E A R L$ (last values of each column) with unknown shift size for $n=\{5,10,15\}, p=\{2,3,4\}, \gamma_{0}=\{0.1,0.2,0.3,0.4,0.5\}$ and $A R L_{0}=370.4$. (D), decreasing case; (I), increasing case; EARL, expected average run length. 


\begin{tabular}{|c|c|c|c|c|}
\hline$\Omega$ & $p$ & $n=5$ & $n=10$ & $n=15$ \\
\hline (D) & 2 & $(1,0.0303,100.9)$ & $\begin{array}{c}\gamma_{0}=0.1 \\
(3,0.0510,47.9)\end{array}$ & $(3,0.0613,32.6)$ \\
\hline (I) & 2 & $(15,0.1625,25.9)$ & $(11,0.1442,15.9)$ & $(10,0.1362,12.1)$ \\
\hline (D) & 3 & $(1,0.0165,135.0)$ & $(2,0.0466,53.1)$ & $(3,0.0576,34.8)$ \\
\hline (I) & 3 & $(17,0.1462,31.1)$ & $(11,0.1379,17.1)$ & $(10,0.1323,12.7)$ \\
\hline (D) & 4 & $(1,0.0033,206.2)$ & $(2,0.0403,59.8)$ & $(3,0.0537,37.3)$ \\
\hline (I) & 4 & $(25,0.1255,41.3)$ & $(12,0.1316,18.5)$ & $(10,0.1283,13.3)$ \\
\hline (D) & 2 & $(1,0.0600,102.1)$ & $\begin{array}{c}\gamma_{0}=0.2 \\
(3,0.1011,48.9)\end{array}$ & $(3,0.1217,33.5)$ \\
\hline (I) & 2 & $(15,0.3325,26.7)$ & $(11,0.2930,16.5)$ & $(9,0.2750,12.6)$ \\
\hline (D) & 3 & $(1,0.0327,136.4)$ & $(2,0.0924,54.2)$ & $(3,0.1142,35.7)$ \\
\hline (I) & 3 & $(17,0.2972,32.0)$ & $(11,0.2795,17.7)$ & $(10,0.2676,13.2)$ \\
\hline (D) & 4 & $(1,0.0066,207.5)$ & $(2,0.0797,61.0)$ & $(3,0.1064,38.3)$ \\
\hline (I) & 4 & $(25,0.2534,42.5)$ & $(12,0.2660,19.2)$ & $(10,0.2590,13.9)$ \\
\hline (D) & 2 & $\left(\begin{array}{llll}1 & 0 & 0889 & 104\end{array}\right)$ & $\begin{array}{c}\gamma_{0}=0.3 \\
(3.0 .1497 .50 .7)\end{array}$ & $(3,0.1804,35.0)$ \\
\hline (I) & 2 & $(14,0.5165,28.0)$ & $(10,0.4497,17.5)$ & $(9,0.4211,13.5)$ \\
\hline (D) & 3 & $(1,0.0484,138.7)$ & $(2,0.1367,56.1)$ & $(3,0.1691,37.2)$ \\
\hline (I) & 3 & $(17,0.4582,33.6)$ & $(11,0.4287,18.9)$ & $(9,0.4074,14.1)$ \\
\hline (D) & 4 & $(1,0.0098,209.7)$ & $(2,0.1178,63.0)$ & $(3,0.1574,39.8)$ \\
\hline (I) & 4 & $(25,0.3859,44.6)$ & $(11,0.4048,20.4)$ & $(10,0.3948,14.8)$ \\
\hline (D) & 2 & $(1,0.1166,106.9)$ & $\begin{array}{c}\gamma_{0}=0.4 \\
(3,0.1962,52.9)\end{array}$ & $(3,0.2368,36.9)$ \\
\hline (I) & 2 & $(14,0.7280,30.1)$ & $(10,0.6219,19.1)$ & $(9,0.5779,14.7)$ \\
\hline (D) & 3 & $(1,0.0633,141.8)$ & $(2,0.1788,58.6)$ & $(3,0.2216,39.2)$ \\
\hline (I) & 3 & $(17,0.6348,36.1)$ & $(11,0.5893,20.5)$ & $(9,0.5569,15.4)$ \\
\hline (D) & 4 & $(1,0.0128,212.7)$ & $(2,0.1539,65.7)$ & $(3,0.2060,41.9)$ \\
\hline (I) & 4 & $(24,0.5235,47.8)$ & $(11,0.5528,22.2)$ & $(9,0.5356,16.2)$ \\
\hline (D) & 2 & $(1,0.1427$, & $\begin{array}{c}\gamma_{0}=0.5 \\
(3,0.2401,55.7)\end{array}$ & $(3,0.2904,39.1)$ \\
\hline (I) & 2 & $(13,0.9746,33.1)$ & $(10,0.8149,21.1)$ & $(9,0.7491,16.2)$ \\
\hline (D) & 3 & $(1,0.0773,145.6)$ & $(2,0.2185,61.5)$ & $(3,0.2714,41.6)$ \\
\hline (I) & 3 & $(16,0.8286,39.8)$ & $(10,0.7615,22.7)$ & $(9,0.7180,17.0)$ \\
\hline (D) & 4 & $(1,0.0155,216.5)$ & $(2,0.1876,69.0)$ & $(3,0.2519,44.5)$ \\
\hline (I) & 4 & $(23,0.6672,52.7)$ & $(11,0.7114,24.7)$ & $(9,0.6869,17.9)$ \\
\hline
\end{tabular}

Table 8. Steady-state performance. Optimal couples $\left(H^{-*}, L C L^{*}\right)$ for $\Omega=[0.5,1)$ and $\left(H^{+*}, U C L^{*}\right)$ for $\Omega=(1,2]$ (two first values of each column) and out-of-control $E A R L$ (last values of each column) with unknown shift size for $n=\{5,10,15\}, p=\{2,3,4\}, \gamma_{0}=\{0.1,0.2,0.3,0.4,0.5\}$ and $A R L_{0}=370.4$. (D), decreasing case; (I), increasing case; EARL, expected average run length. 


\begin{tabular}{|c|c|c|c|c|c|c|c|c|c|}
\hline \multirow[b]{2}{*}{$\Omega$} & \multicolumn{3}{|c|}{$p=2$} & \multicolumn{3}{|c|}{$p=3$} & \multicolumn{3}{|c|}{$p=4$} \\
\hline & $n=5$ & $n=10$ & $n=15$ & $n=5$ & $n=10$ & $n=15$ & $n=5$ & $n=10$ & $n=15$ \\
\hline & & & & & $\gamma_{0}=0$ & & & & \\
\hline (D) & 40 & 45 & 46 & 35 & 44 & 46 & 24 & 44 & 46 \\
\hline (I) & 36 & 40 & 42 & 34 & 39 & 41 & 32 & 39 & 41 \\
\hline & & & & & $\gamma_{0}=0$ & & & & \\
\hline (D) & 39 & 44 & 46 & 35 & 44 & 46 & 24 & 43 & 45 \\
\hline (I) & 36 & 40 & 42 & 34 & 39 & 41 & 32 & 39 & 41 \\
\hline & & & & & $\gamma_{0}=0$ & & & & \\
\hline (D) & 39 & 44 & 45 & 35 & 43 & 45 & 24 & 43 & 45 \\
\hline (I) & 36 & 40 & 41 & 35 & 39 & 41 & 32 & 39 & 41 \\
\hline & & & & & $\gamma_{0}=0$ & & & & \\
\hline (D) & 38 & 43 & 45 & 34 & 43 & 45 & 23 & 42 & 44 \\
\hline (I) & 36 & 40 & 41 & 35 & 39 & 41 & 32 & 39 & 41 \\
\hline & & & & & $\gamma_{0}=0$ & & & & \\
\hline (D) & 38 & 43 & 44 & 33 & 42 & 44 & 23 & 42 & 44 \\
\hline (I) & 37 & 40 & 42 & 36 & 40 & 41 & 33 & 39 & 41 \\
\hline
\end{tabular}

Table 9. Comparison vs. the Shewhart MCV control chart. $\Delta_{E}$ values of the Synthetic MCV control charts with unknown shift for different values of $p=\{2,3,4\}, n=\{5,10,15\}, \gamma_{0}=\{0.1,0.2,0.3,0.4,0.5\}$ and $A R L_{0}=$ 370.4 . 


\begin{tabular}{|c|c|c|c|c|c|c|c|c|c|}
\hline \multirow{4}{*}{$p$} & \multirow{4}{*}{$\tau$} & \multicolumn{4}{|c|}{$n=5$} & \multicolumn{4}{|c|}{$n=10$} \\
\hline & & \multicolumn{2}{|c|}{ zero-state } & \multicolumn{2}{|c|}{ steady-state } & \multicolumn{2}{|c|}{ zero-state } & \multicolumn{2}{|c|}{ steady-state } \\
\hline & & Syn MCV & RS MCV & Syn MCV & RS MCV & Syn MCV & RS MCV & Syn MCV & RS MCV \\
\hline & & & & & & & & & \\
\hline \multirow[t]{6}{*}{2} & 0.50 & 10.6 & 6.4 & 13.5 & 4.3 & 1.5 & 2.6 & 2.7 & 2.0 \\
\hline & 0.75 & 77.2 & 28.9 & 82.8 & 24.7 & 16.3 & 10.6 & 21.4 & 7.8 \\
\hline & 0.90 & 206.3 & 122.1 & 210.8 & 118.2 & 105.3 & 61.0 & 115.4 & 57.0 \\
\hline & 1.10 & 74.7 & 88.3 & 94.0 & 86.1 & 44.1 & 48.9 & 58.6 & 47.3 \\
\hline & 1.25 & 17.9 & 23.8 & 27.1 & 22.0 & 8.1 & 10.5 & 13.1 & 9.0 \\
\hline & 1.50 & 5.4 & 7.8 & 9.2 & 6.8 & 2.4 & 3.6 & 4.4 & 3.0 \\
\hline \multirow[t]{7}{*}{3} & 0.50 & 27.3 & 9.6 & 30.9 & 6.3 & 1.8 & 3.0 & 3.1 & 2.2 \\
\hline & 0.75 & 122.6 & 43.7 & 127.0 & 39.3 & 20.6 & 12.1 & 26.2 & 9.0 \\
\hline & 0.90 & 246.3 & 153.8 & 249.1 & 150.4 & 117.9 & 68.1 & 127.5 & 64.0 \\
\hline & 1.10 & 88.4 & 107.1 & 109.3 & 105.0 & 47.9 & 53.7 & 63.2 & 51.5 \\
\hline & 1.25 & 23.7 & 32.3 & 34.9 & 30.7 & 9.1 & 11.8 & 14.6 & 10.2 \\
\hline & 1.50 & 7.4 & 10.8 & 12.4 & 9.8 & 2.7 & 4.0 & 4.8 & 3.3 \\
\hline & & \multicolumn{8}{|c|}{$\gamma_{0}=\overline{0.3}$} \\
\hline \multirow[t]{6}{*}{2} & 0.50 & 11.4 & 6.7 & 14.4 & 4.4 & 1.6 & 2.8 & 2.9 & 2.1 \\
\hline & 0.75 & 81.3 & 30.7 & 87.0 & 26.5 & 18.3 & 11.6 & 23.7 & 8.5 \\
\hline & 0.90 & 211.3 & 127.2 & 215.6 & 123.5 & 112.1 & 66.1 & 122.2 & 62.0 \\
\hline & 1.10 & 80.4 & 94.4 & 100.0 & 92.2 & 49.4 & 54.7 & 64.7 & 52.5 \\
\hline & 1.25 & 20.2 & 26.6 & 30.2 & 24.8 & 9.6 & 12.1 & 15.2 & 10.6 \\
\hline & 1.50 & 6.2 & 8.9 & 10.5 & 7.8 & 2.8 & 4.2 & 5.1 & 3.5 \\
\hline \multirow[t]{7}{*}{3} & 0.50 & 29.0 & 10.0 & 32.7 & 6.6 & 1.9 & 3.1 & 3.4 & 2.3 \\
\hline & 0.75 & 127.4 & 46.4 & 131.8 & 42.1 & 23.1 & 13.1 & 28.9 & 9.9 \\
\hline & 0.90 & 250.5 & 159.3 & 253.2 & 156.0 & 124.9 & 73.4 & 134.5 & 69.3 \\
\hline & 1.10 & 94.5 & 113.3 & 115.7 & 112.1 & 53.6 & 60.1 & 69.6 & 57.9 \\
\hline & 1.25 & 26.6 & 35.9 & 38.6 & 34.4 & 10.8 & 13.7 & 17.0 & 12.0 \\
\hline & 1.50 & 8.6 & 12.5 & 14.2 & 11.4 & 3.2 & 4.7 & 5.7 & 3.9 \\
\hline & & \multicolumn{8}{|c|}{$\gamma_{0}=\overline{0.5}$} \\
\hline \multirow[t]{6}{*}{2} & 0.50 & 13.2 & 7.2 & 16.3 & 4.83 & 1.8 & 3.0 & 3.2 & 2.2 \\
\hline & 0.75 & 89.2 & 34.7 & 94.9 & 30.4 & 22.2 & 13.3 & 28.2 & 10.1 \\
\hline & 0.90 & 220.0 & 137.3 & 224.2 & 133.6 & 123.8 & 75.5 & 134.0 & 71.4 \\
\hline & 1.10 & 94.0 & 108.2 & 114.0 & 106.9 & 60.8 & 66.6 & 77.2 & 64.4 \\
\hline & 1.25 & 26.3 & 33.5 & 37.8 & 31.8 & 13.0 & 15.9 & 20.0 & 14.1 \\
\hline & 1.50 & 8.5 & 11.9 & 13.9 & 10.6 & 3.9 & 5.5 & 6.7 & 4.6 \\
\hline \multirow[t]{6}{*}{3} & 0.50 & 32.6 & 10.7 & 36.4 & 7.3 & 2.3 & 3.4 & 3.8 & 2.5 \\
\hline & 0.75 & 136.5 & 52.1 & 140.8 & 47.7 & 27.8 & 15.2 & 34.3 & 11.9 \\
\hline & 0.90 & 258.0 & 169.7 & 260.6 & 166.5 & 137.1 & 83.4 & 146.6 & 79.3 \\
\hline & 1.10 & 109.2 & 127.7 & 130.4 & 126.5 & 65.6 & 72.0 & 82.7 & 70.5 \\
\hline & 1.25 & 34.3 & 44.9 & 47.9 & 43.2 & 14.7 & 17.8 & 22.3 & 16.1 \\
\hline & 1.50 & 11.9 & 17.0 & 18.9 & 15.6 & 4.4 & 6.2 & 7.6 & 5.2 \\
\hline
\end{tabular}

Table 10. Comparison with the run sum control charts, for different values of $n=\{5,10\}, p=\{2,3\}$, $\gamma_{0}=\{0.1,0.2,0.3\}, \tau=\{0.5,0.75,0.9,1.10,1.25,1.50\}$ and $A R L_{0}=370$.

\begin{tabular}{|c|c|c|c|c|c|c|c|c|c|}
\hline \multirow{3}{*}{$p$} & \multirow{3}{*}{$\tau$} & \multicolumn{4}{|c|}{$n=5$} & \multicolumn{4}{|c|}{$n=10$} \\
\hline & & Syn MCV & VSSI MCV & VSI MCV & VSS MCV & Syn MCV & VSSI MCV & VSI MCV & VSS MCV \\
\hline & & \multicolumn{8}{|c|}{$\gamma_{0}=\overline{0.1}$} \\
\hline \multirow[t]{2}{*}{2} & 1.25 & 27.08 & 16.99 & 31.48 & 19.29 & 13.10 & 5.79 & 11.84 & 8.80 \\
\hline & 1.50 & 9.19 & 4.19 & 7.32 & 5.33 & 4.36 & 1.66 & 1.74 & 2.70 \\
\hline \multirow[t]{3}{*}{3} & 1.25 & 34.92 & 26.37 & 41.82 & 28.28 & 14.61 & 6.79 & 13.98 & 9.83 \\
\hline & 1.50 & 12.40 & 6.28 & 11.82 & 7.29 & 4.82 & 1.86 & 1.96 & 2.92 \\
\hline & & \multicolumn{8}{|c|}{$\gamma_{0}=\overline{0.3}$} \\
\hline \multirow[t]{2}{*}{2} & 1.25 & 30.18 & 20.88 & 35.69 & 23.54 & 15.24 & 7.30 & 14.49 & 10.95 \\
\hline & 1.50 & 10.53 & 4.94 & 9.25 & 6.08 & 5.08 & 1.93 & 2.03 & 3.08 \\
\hline \multirow[t]{3}{*}{3} & 1.25 & 38.67 & 31.93 & 46.81 & 34.06 & 16.97 & 8.64 & 17.01 & 12.29 \\
\hline & 1.50 & 14.20 & 7.55 & 14.42 & 8.55 & 5.65 & 2.21 & 2.37 & 3.36 \\
\hline & & \multicolumn{8}{|c|}{$\overline{\gamma_{0}}=\overline{0.5}$} \\
\hline \multirow[t]{2}{*}{2} & 1.25 & 37.77 & 30.62 & 46.94 & 34.02 & 19.98 & 11.18 & 20.74 & 16.14 \\
\hline & 1.50 & 13.93 & 6.79 & 14.81 & 8.09 & 6.74 & 2.60 & 4.57 & 4.05 \\
\hline \multirow[t]{2}{*}{3} & 1.25 & 47.97 & 45.14 & 60.17 & 47.68 & 22.27 & 13.33 & 24.16 & 18.24 \\
\hline & 1.50 & 18.92 & 10.81 & 21.90 & 11.99 & 7.58 & 3.00 & 5.79 & 4.48 \\
\hline
\end{tabular}

Table 11. Comparison with the other adaptive control charts proposed in [29] under the steady-state condition, for different values of $n=\{5,10\}, p=\{2,3\}, \gamma_{0}=\{0.1,0.2,0.3\}, A R L_{0}=370.4$ and upper shifts $\tau=\{1.25,1.50\}$ 